\title{
Are black holes a serious threat to scalar field dark matter models?
}

\author{
Juan Barranco,, , * Argelia Bernal, $2,+$ Juan Carlos Degollado, $1, \pm$ Alberto Diez-Tejedor, 2,8 \\ Miguel Megevand, ${ }^{2}, \uparrow$ Miguel Alcubierre, $2, * *$ Darío Núnez, ${ }^{2,+\dagger}$ and Olivier Sarbach $3, \pm$
}

(Part of the "Instituto Avanzado de Cosmología" collaboration)

${ }^{1}$ Instituto de Astronomía, Universidad Nacional Autónoma de México,

Circuito Exterior C.U., A.P. 70-264, México D.F. 04510, México

${ }^{2}$ Instituto de Ciencias Nucleares, Universidad Nacional Autónoma de México,

Circuito Exterior C.U., A.P. 70-543, México D.F. 04510, México

${ }^{3}$ Instituto de Física y Matemáticas,

Universidad Michoacana de San Nicolás de Hidalgo, Edificio C-3,

Ciudad Universitaria, 58040 Morelia, Michoacán, México

(Dated: August 5, 2011)

\begin{abstract}
Classical scalar fields have been proposed as possible candidates for the dark matter component of the universe. Given the fact that super-massive black holes seem to exist at the center of most galaxies, in order to be a viable candidate for the dark matter halo a scalar field configuration should be stable in the presence of a central black hole, or at least be able to survive for cosmological timescales. In the present work we consider a scalar field as a test field on a Schwarzschild background, and study under which conditions one can obtain long-lived configurations. We present a detailed study of the Klein-Gordon equation in the Schwarzschild spacetime, both from an analytical and numerical point of view, and show that indeed there exist quasi-stationary solutions that can remain surrounding a black hole for large time-scales.
\end{abstract}

PACS numbers: 95.30.Sf 95.35.+d 98.80.Jk 98.62.Mw

\footnotetext{
* barranco@astroscu.unam.mx

$\dagger$ argelia.bernal@nucleares.unam.mx

$\ddagger$ jdaza@astroscu.unam.mx

$\S$ alberto.diez@nucleares.unam.mx

ฯ megevand@nucleares.unam.mx

** malcubi@nucleares.unam.mx

†† nunez@nucleares.unam.mx


} 


\section{INTRODUCTION}

Scalar fields are a common theme in modern cosmology. They play a central role in inflation [1], and they have been frequently used to describe dark energy in place of the cosmological constant [2, 3]. Moreover, even if the usual description of dark matter is done in terms of weakly interacting massive particles (WIMPs), given the persistent uncertainty about the real nature of that mysterious component, one can not a priori reject one candidate in favor of another. In this context, ultra-light scalar fields have also been invoked as possible alternative dark matter candidates [4 21$]$.

In modern physics quantum fields are considered to be the fundamental constituents of nature. For the particular case of a boson field which has not been in thermal equilibrium with the other fields of the standard model of particle physics, a classical field description (i.e. a coherent excitation) seems in principle as natural as that given by the WIMP hypothesis. Indeed, a description in terms of classical scalar fields has been shown to describe the large scale cosmological scenario (to linear order) as consistently as cold dark matter (CDM) [11, 16, 22, 23]. In a more local context, massive complex scalar fields can form spherically symmetric, asymptotically flat, regular, self-gravitating configurations known as "boson stars" [24, 25] (see also [26, 27] and references therein). For the case of a scalar field with a mass of the order of $10^{-22}-10^{-24} \mathrm{eV}$, these configurations can have the mass and the size expected for a typical galactic halo [5, 13, 28]. However, it has been recognized that even though such configurations are stable [29, 30], they do not reproduce the observed velocity profiles (though this problem can be significantly alleviated with the use of multi-state configurations, see e.g. [31 33]). Finally, ultra-light scalar field dark matter (SFDM) could have some advantages over the standard Lambda cold dark matter $(\Lambda \mathrm{CDM})$ scenario, namely that the SFDM model is not expected to produce an over-density of satellite galaxies [11], nor

does it result in the generation of the cuspy halos typically obtained in the high-resolution N-body simulations performed within the standard $\Lambda$ CDM model [14, 15, 18, 21].

Despite all this, there has been some controversy regarding the possibility that dark matter halos could be described by coherent scalar excitations. The central issue being the fact that most galaxies seem to host a super-massive black hole at their centers, so that a scalar field halo should be able to survive surrounding such a black hole for cosmological timescales. Regarding that question, it has been known for a long time that static, spherically 
symmetric, asymptotically flat scalar field configurations with a Schwarzschild black hole at their center can not exist in nature [34], i.e. a Schwarzschild black hole can not have "scalar hair" [35]. 1 However, these "no-hair" theorems have nothing to say about a specific time-scale, and it could in principle be possible that scalar field configurations could survive the birth of a super-massive black hole and remain in a quasi-stationary state for very long times.

The question of how a scalar field is accreted into a Schwarzschild background has attracted the attention of many researchers, motivated in some cases by the fact that dark energy can be modeled by a scalar field [37-43], or because of the scalar dark matter models [44, 45]. A definite picture of the accretion of a scalar field onto a black hole is not clear yet and there is in fact some controversy around it. For instance, in [44] it is argued that the accretion rate will be very slow, while others argue that this accretion will be fast [45]. Very long lasting configurations have also been found using "artificial" scalar field potentials [46].

Here we will restrict ourselves to the case of a scalar field as a test field in the background spacetime of a Schwarzschild black hole. It is for this reason that we will talk about "scalar field clouds" rather than "galactic halos", in order to maintain a clear perspective of the real problem we are studying. The dark matter content in the central region of a galactic halo is very diluted, so it is to be expected that this approximation could shed some light on the nature of the dark matter problem. However, we would like to emphasize that this is only a first step on the study of long-lived scalar field configurations surrounding a black hole, and the self-gravitating case will be considered in future works.

This paper can be separated in two distinct parts plus the conclusions. In a first part (Section II), we introduce the problem and present some important analytical results. We find that it is indeed not possible to obtain a stationary, localized, physical scalar field configuration surrounding a Schwarzschild black hole: Solutions that are well behaved at spatial infinity are not regular at the horizon; they oscillate with a divergent frequency and these oscillations produce an infinite integrated energy close to the horizon. Thus, in particular, such solutions are not compatible with the test field limit approximation. This is of course not surprising if we remember the no-hair theorems. However, these non-physical solutions can be quite useful in order to construct appropriate initial data for a special set

\footnotetext{
${ }^{1}$ Here we will be working within the domain of general relativity. However, "hairy" black hole configurations are indeed possible in the context of scalar-tensor theories of gravity [36].
} 
of physical quasi-stationary (resonant) scalar field configurations with very long lifetimes. In a second part (Section III), we perform numerical evolutions in order to determine the lifetime of such quasi-stationary resonant configurations. Finally, we discuss our findings in Section IV.

Throughout the paper we will use geometric units such that $G=c=1$, and we will use the conventions of Misner, Thorne and Wheeler (MTW) [47]. In particular, the signature of the spacetime metric will be taken to be $(-,+,+,+)$.

\section{EIGENMODE ANALYSIS AND RESONANCES}

We will be looking for stationary scalar field configurations surrounding a Schwarzschild black hole. Here we will restrict ourselves to the case of a canonical, massive, non-selfinteracting, minimally coupled scalar field $\phi$. The equation of motion for that field is given by the Klein-Gordon equation

$$
\left(\square-\mu^{2}\right) \phi=0
$$

with the d'Alambertian operator defined as $\square:=(1 / \sqrt{-g}) \partial_{\mu}\left(\sqrt{-g} g^{\mu \nu} \partial_{\nu}\right)$. With our conventions $\phi$ is dimensionless, while $\mu$ has dimensions of (length) ${ }^{-1}$. The associated quantum mechanical "mass" of the scalar field is given by $\hbar \mu$, but all our considerations in this work will be purely classical. (For the SFDM models usually considered in the literature one usually has $\hbar \mu \sim 10^{-22}-10^{-24} \mathrm{eV}$ in physical units, which implies $\mu$ of the order of $1-10^{-2}(\mathrm{pc})^{-1}$.)

We will also be assuming that the energy associated with the scalar field configuration is very small compared to the mass of the black hole, so that the gravitational back-reaction associated to the scalar field distribution can be disregarded. Under this assumption, and working in Schwarzschild coordinates, we can write the spacetime metric as

$$
d s^{2}=-N(r) d t^{2}+\frac{d r^{2}}{N(r)}+r^{2} d \Omega^{2}, \quad N(r):=1-2 M / r
$$

with $M$ the mass of the black hole and $d \Omega^{2}:=d \theta^{2}+\sin ^{2} \theta d \varphi^{2}$ the standard solid angle

element. This coordinate system is suitable to describe the physics in the exterior region $r \in(2 M, \infty)$.

To look for solutions of the Klein-Gordon equation above, we will start by considering a 
decomposition into spherical harmonics: 2

$$
\phi(t, r, \theta, \varphi)=\frac{1}{r} \sum_{\ell, m} \psi_{\ell m}(t, r) Y^{\ell m}(\theta, \varphi),
$$

where the $1 / r$ factor has been introduced for convenience, and the parameters $\ell$ and $m$ take the usual values: $\ell \in\{0,1,2, \ldots\},-\ell \leq m \leq \ell$. Substituting the above equation into (11) we obtain the following family of reduced equations

$$
\left[\frac{1}{N(r)} \frac{\partial^{2}}{\partial t^{2}}-\frac{\partial}{\partial r} N(r) \frac{\partial}{\partial r}+\mathcal{U}_{\ell}(\mu, M ; r)\right] \psi_{\ell m}=0
$$

where we have defined

$$
\mathcal{U}_{\ell}(\mu, M ; r):=\frac{\ell(\ell+1)}{r^{2}}+\frac{2 M}{r^{3}}+\mu^{2} .
$$

Notice that $\mathcal{U}_{\ell}$ is an everywhere positive and monotonically decreasing function of $r$, and also that equation (44) does not depend on $m$. The first term in (5) is the usual centrifugal term, the second one is a curvature correction, and the third one is inherited from the corresponding term in the Klein-Gordon equation, Eq. (11).

In the test field limit all configurations have the conserved energy $E=\sum_{\ell, m} E_{\ell m}$, with

$$
E_{\ell m}=\int_{2 M}^{\infty} \rho_{E}(r) d r
$$

and where

$$
\rho_{E}(r)=\frac{1}{2}\left(\frac{1}{N(r)}\left|\frac{\partial \psi_{\ell m}}{\partial t}\right|^{2}+N(r)\left|\frac{\partial \psi_{\ell m}}{\partial r}\right|^{2}+\mathcal{U}_{\ell}(\mu, M ; r)\left|\psi_{\ell m}\right|^{2}\right)
$$

is the energy density integrated over the sphere as a function of areal radius. In what follows we will refer to this integrated energy density simply as the "radial energy density".

To obtain the last expression we have used the fact that it is always possible to associate a conserved charge $Q_{a}=\int_{\Sigma} k_{a}{ }^{\mu} T_{\mu \nu} n^{\mu} \sqrt{\gamma} d \Sigma$ to every Killing vector field $k_{a}$. Here $n^{\mu}$ is a time-like, future-directed, normalized 4-vector orthogonal to the 3-dimensional space-like hypersurface $\Sigma$, and $\sqrt{\gamma} d \Sigma$ is the volume element on $\Sigma$. The subindex $a$ labels the different Killing fields associated with the background metric, and $T_{\mu \nu}$ is the energy-momentum tensor

\footnotetext{
${ }^{2}$ If the scalar field was real we would have $\psi_{\ell,-m}=(-1)^{m} \psi_{\ell, m}^{*}$, i.e. not all the modes $\psi_{\ell m}(t, r)$ in (3) are independent of each other. Apart from this, all the results obtained below apply for the real as well as the complex cases. The differences between the real and complex scalar field will appear when considering the energy-momentum tensor, but this is not relevant for the test field limit approximation we are considering here.
} 
for the matter fields. Here we will be only interested in those configurations with a finite value for the integrals above. Under this assumption and the additional requirement of $\Sigma$ being a Cauchy surface, it follows that the charges $Q_{a}$ do not depend on the particular choice of $\Sigma$; in particular the charges are preserved under time evolution. For the case $k_{0}=\partial_{t}$ and $\Sigma=\{t=$ const. $\}$, where $(t, r, \theta, \varphi)$ are the usual Schwarzschild coordinates, we arrive at expressions (6). The hypersurfaces $\Sigma$ are Cauchy surfaces for the Schwarzschild exterior spacetime, and as a consequence the energy (6) is conserved. However, in the numerical simulations of Section [II below, we will be integrating the energy density over a different foliation which extends from the future horizon to some finite, large radius, and the resulting energy will, in fact, decay due to energy loses through the horizon and the outer boundary.

\section{A. Reduction to a time-independent Schrödinger-like problem: mode solutions}

In order to look for the stationary solutions of Eq. (41) we make a further decomposition of the functions $\psi_{\ell m}(t, r)$ into oscillating modes of the form:

$$
\psi_{\ell m}(t, r)=e^{i \omega_{\ell m} t} u_{\ell m}(r)
$$

with $\omega_{\ell m}$ a real frequency, and $u_{\ell m}(r)$ a complex function of $r$ in the interval $(2 M, \infty)$. In order to simplify the notation from now on we will omit the labels $\ell$ and $m$ from $\omega_{\ell m}$ and $u_{\ell m}$.

Introducing the ansatz (7) into equation (44) one finds the following eigenvalue problem

$$
\left[-N(r) \frac{\partial}{\partial r}\left(N(r) \frac{\partial}{\partial r}\right)+N(r) \mathcal{U}_{\ell}(\mu, M ; r)\right] u(r)=\omega^{2} u(r), \quad 2 M<r<\infty .
$$

After defining the Regge-Wheeler tortoise coordinate $r^{*}:=r+2 M \ln (r / 2 M-1)$, this equation can be rewritten as the following time-independent Schrödinger-like equation:

$$
\left[-\frac{\partial^{2}}{\partial r^{* 2}}+V_{\text {eff }}\left(r^{*}\right)\right] u\left(r^{*}\right)=\omega^{2} u\left(r^{*}\right), \quad-\infty<r^{*}<\infty,
$$

with the "effective potential" $V_{\text {eff }}\left(r^{*}\right)$ defined as

$$
V_{\mathrm{eff}}\left(r^{*}\right):=N(r) \mathcal{U}_{\ell}(\mu, M ; r), \quad r=r\left(r^{*}\right)
$$

Notice that in the usual non-relativistic quantum mechanical case one would have $\omega$ instead

of $\omega^{2}$ in Eq. (9). This is because the Schrödinger equation is first order in time, whereas the 


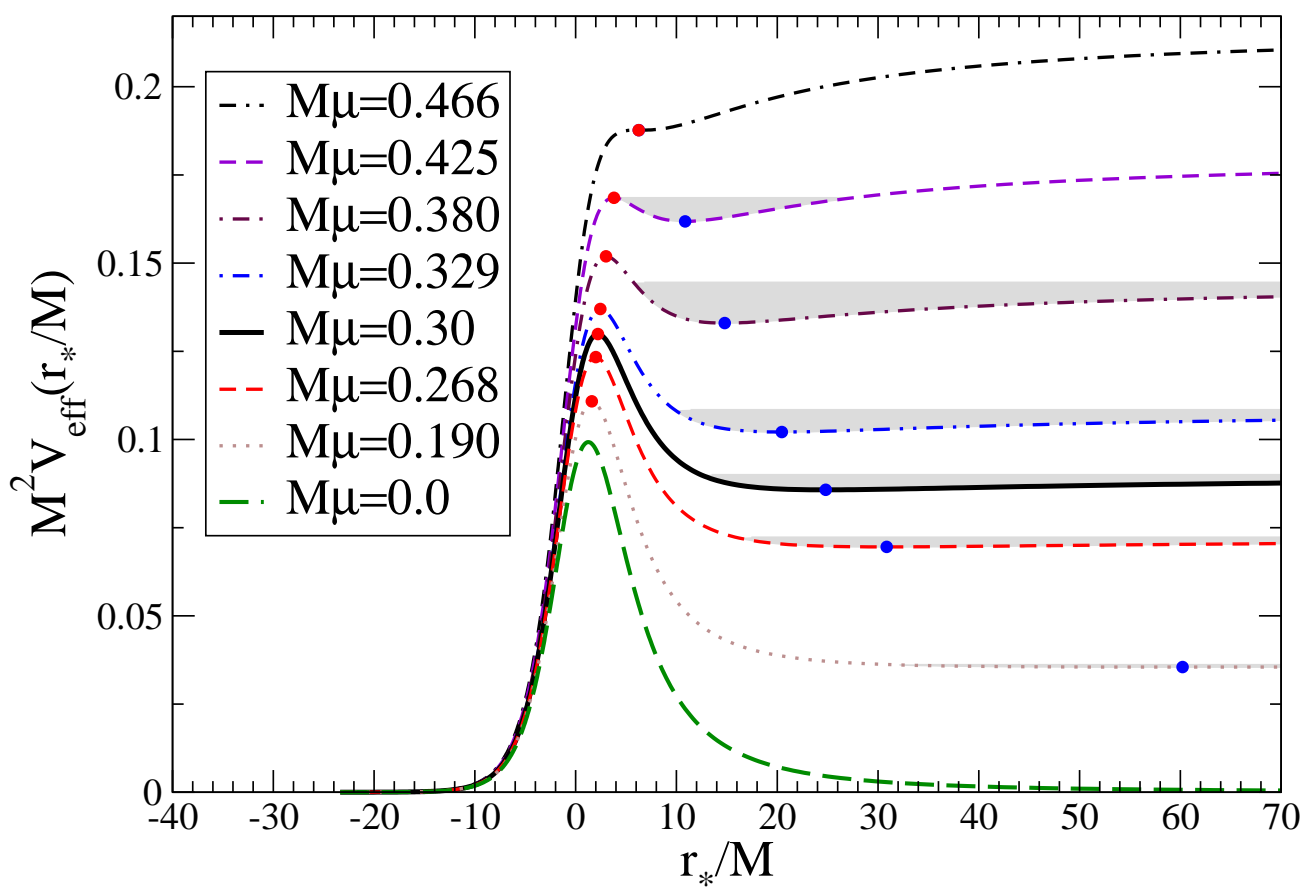

FIG. 1. The effective potential $M^{2} V_{\text {eff }}$ for $\ell=1$, and different values of the parameter $M \mu$. The shaded regions represent the "resonance bands" where the potential has a local minimum (see Section $\llbracket \mathrm{IC}$ below). Notice that for $M \mu=0$ and $M \mu \gtrsim 0.466$ the local minimum does not appear. For the case $M \mu=0$ we recover the usual Regge-Wheeler potential. The case with $M \mu=0.3$ will be considered later on in our future examples and it appears as a solid line to emphasize it.

Klein-Gordon equation is second order. Also, note that equation (9) in fact only depends on the parameters $\ell, M \mu$ and $M \omega$. Since that equation is invariant under $\omega \rightarrow-\omega$ we can restrict ourselves to the case $\omega>0$.

The typical form of the effective potential can be seen in Fig. 1. Contrary to what happens in the usual Regge-Wheeler equation, the asymptotic value of $V_{\text {eff }}$ for large radii is not zero but $\mu^{2}$. This fact will turn out to be crucial in the study of the behavior of the solutions to equation (9).

In the following, we will analyze the spectrum of the Schrödinger operator given above in more detail. The operator can be separated into the point and the continuous spectrum. The point spectrum consists of the eigenvalues of Eq. (9) for which the square norm

$$
\|u\|^{2}:=\int_{2 M}^{\infty}\left(N(r)\left|\frac{\partial u}{\partial r}\right|^{2}+\mathcal{U}_{\ell}(\mu, M ; r)|u|^{2}\right) d r
$$


is finite. Such solutions, when they exist, describe bound states in the quantum mechanical picture. In the context of the Klein-Gordon equation they will give rise to stationary modes $\psi(t, r)=e^{i \omega t} u(r)$ with finite energy. However, as we will show below, such solutions do not exist in our case.

This leaves us with the continuous spectrum consisting on the values of $\omega^{2}$ for which the time-independent Schrödinger equation (9) admits solutions which do not grow faster than a polynomial as $r^{*} \rightarrow \pm \infty$. These solutions describe scattering states and will be analyzed in more detail below.

At this point it is important to mention that exact solutions to equation (9) are in fact known and can be expressed in terms of the so-called "Heun functions" (see e.g. [48, 49]). However, we will not use such exact solutions here and will rather concentrate on the general behavior of the solutions.

We will start with an analysis of the asymptotic behavior of the solutions to Eq. (99), both close to the horizon and close to infinity.

\section{B. Asymptotic behavior and spectrum}

Regarding the behavior of the effective potential notice first that, as already mentioned above, in the limit $r^{*} \rightarrow \infty$ it approaches the constant value $\mu^{2}$, so that Eq. (9) reduces to

$$
\frac{\partial^{2} u}{\partial r^{* 2}}+k^{2} u=0, \quad k^{2}:=\omega^{2}-\mu^{2} .
$$

The general solution to this equation is a linear combination of the functions $u=e^{ \pm i k r^{*}}$, with

$k:=\sqrt{\omega^{2}-\mu^{2}}$. Therefore, as long as $0<\omega^{2}<\mu^{2}$ the parameter $k$ will be purely imaginary and we will have one exponentially decaying and one exponentially growing solution, while for $\omega^{2}>\mu^{2}$ the parameter $k$ will be real and both solutions will be oscillatory.

For $r^{*} \rightarrow-\infty$, on the other hand, the effective potential $V_{\text {eff }}$ decays exponentially fast to zero since $N(r)=2 M r^{-1}(r / 2 M-1)=2 M r^{-1} \exp \left(\left(r^{*}-r\right) / 2 M\right)$. Therefore, the solutions are oscillatory for all $\omega^{2}>0$ (notice that we are assuming $\omega$ to be real so this is always the case), and they can be expressed as a linear combination of the functions $u=e^{ \pm i \omega r^{*}}$.

Consider now the interval $0<\omega^{2}<\mu^{2}$. For $\omega^{2}$ to belong to the spectrum, the right asymptotic solution must be proportional to $e^{-|k| r^{*}}$, and the left asymptotic solution may 
be a linear combination of $e^{ \pm i \omega r^{*}}$ of the form

$$
u \propto e^{-i \omega r^{*}}+R e^{i \omega r^{*}}
$$

with $R$ a reflection coefficient. However, since the right asymptotic solution and the coefficients in Eq. (9) are real, so must be the left asymptotic solution, which implies that $|R|=1$ such that $u \propto \cos \left(\omega r^{*}-\delta\right)$ for some phase factor $\delta$. In particular, the solution is not normalizable with respect to the square norm given in (11), and so it does not give rise to a bound state. Therefore, the spectrum for $0<\omega^{2}<\mu^{2}$ is continuous and non-degenerate. From the point of view of scattering theory, $|R|=1$ simply means that a plane wave which travels from $r^{*} \rightarrow-\infty$ to the right with frequency $0<\omega^{2}<\mu^{2}$ is completely reflected from the potential since it does not have enough "energy" to reach $r^{*} \rightarrow+\infty$. For the Klein-Gordon equation, this means that the corresponding stationary mode solution satisfies $\psi(t, r) \propto e^{i \omega\left(t-r^{*}\right)}+R e^{i \omega\left(t+r^{*}\right)}$ for $r^{*} \rightarrow-\infty$. Since $R \neq 0$, the solution is always singular at both the future and past horizons.

Finally, for $\omega^{2}>\mu^{2}$ the asymptotic solutions at $r^{*} \rightarrow \pm \infty$ are both oscillatory, and the spectrum is continuous and degenerate since there are two linearly independent solutions which are bounded at $r^{*} \rightarrow \pm \infty$.

Notice that the mode solutions with $0<\omega^{2}<\mu^{2}$ do not have a finite value for the energy, expression (6), but they are still "localized" around the black hole since they decay exponentially at large $r^{*}$. This is not the case for the mode solutions with $\omega^{2}>\mu^{2}$, which do not decay at spatial infinity.

\section{Resonant States}

We will now take a closer look at the energy interval $0<\omega^{2}<\mu^{2}$. Even though the reflection coefficient is one in this case (see Eq. (13) above), it is still possible to have resonances if $V_{\text {eff }}\left(r^{*}\right)$ has a "potential well" with a local minimum lying below $\mu^{2}$. In this case, plane waves emanating from $r^{*} \rightarrow-\infty$ may tunnel through the potential barrier into the potential well, and if they have the correct frequency their amplitude may build up through multiple reflections at the walls of the potential well. As we will show below, such resonances indeed occur for a discrete set of energy levels.

Clearly, a necessary condition for this phenomena to take place is the existence of the 
potential well. In order to find under which conditions such a potential well exists we first need to determine the critical points of the effective potential $V_{\text {eff. }}$. It is in fact not difficult to show that such critical points correspond to the roots of the following third degree polynomial in $r$ (notice that here we are working with $r$ and not with $r^{*}$ ):

$$
M \mu^{2} r^{3}-\ell(\ell+1) r^{2}+3 M\left(\ell^{2}+\ell-1\right) r+8 M^{2}=0 .
$$

This polynomial can be shown to have three real solutions if the following condition is verified:

$$
\begin{aligned}
(M \mu)^{2} & <-\frac{1}{32}\left(\ell^{2}+\ell-1\right)\left(\ell^{2}+\ell+1\right)^{2} \\
& +\frac{1}{288} \sqrt{3\left(3 \ell^{4}+6 \ell^{3}+5 \ell^{2}+2 \ell+3\right)^{3}},
\end{aligned}
$$

If $M \mu$ satisfies relation (15) above, the potential $V_{\text {eff }}$ will have three critical points. One of them always corresponds to a negative value of $r$, so it is not of interest to our problem (negative values of $r$ are unphysical). The other two critical points correspond to a minimum and a maximum of the effective potential.

For the case $\mu=0$ we recover the well-known solutions for which the maximum of the potential is located at:

$$
r_{\max }=\frac{3 M}{2}\left(1+\sqrt{1+\frac{14 \ell^{2}+14 \ell+9}{9 \ell^{2}(\ell+1)^{2}}}-\frac{1}{2 \ell(\ell+1)}\right) .
$$

For $\ell \rightarrow \infty$ the maximum occurs at $r_{\max }=3 M$, which represents the innermost stable circular orbit for massless classical particles. Any particle that starts with vanishing radial velocity in the region $r<3 M$ will fall into the black hole.

The region in parameter space where a potential well exists and resonances are possible is illustrated in Fig. 2 for different values of $\ell$ (see also Fig. 1). We will call this region the "resonance band", $V_{\text {eff }}^{\min }<\omega^{2}<\min \left\{\mathrm{V}_{\text {eff }}^{\max }, \mu^{2}\right\}$, although not all the states in that band will be resonant. Here $V_{\text {eff }}^{\min }$ and $V_{\text {eff }}^{\max }$ are the local minimum and maximum, respectively, represented with dots in Fig. 1. The cut-off in $M \mu$ that appears for each value of $\ell$ corresponds to a value of $M \mu$ large enough so that relation (15) is no longer satisfied and the local minimum of the potential does not appear. Notice that such a cut-off increases with $\ell$, so that for a mass of the scalar field such that $M \mu<1 / 4$ the effective potential will have a minimum for all possible values of $\ell$. 3 We also want to stress the fact that we can have a

${ }^{3}$ Taking the mass of a typical black hole in the center of a galaxy to be of the order $M \sim 10^{8} M_{\odot}$ we obtain that the mass of the scalar field should be less than $10^{-18} \mathrm{eV}$. Compare that result with the expected mass for the axion, $10^{-5}$ to $10^{-3} \mathrm{eV}\left[\underline{50}\right.$ ], and with the mass of the SFDM models, $10^{-22}$ to $10^{-24} \mathrm{eV}$. 


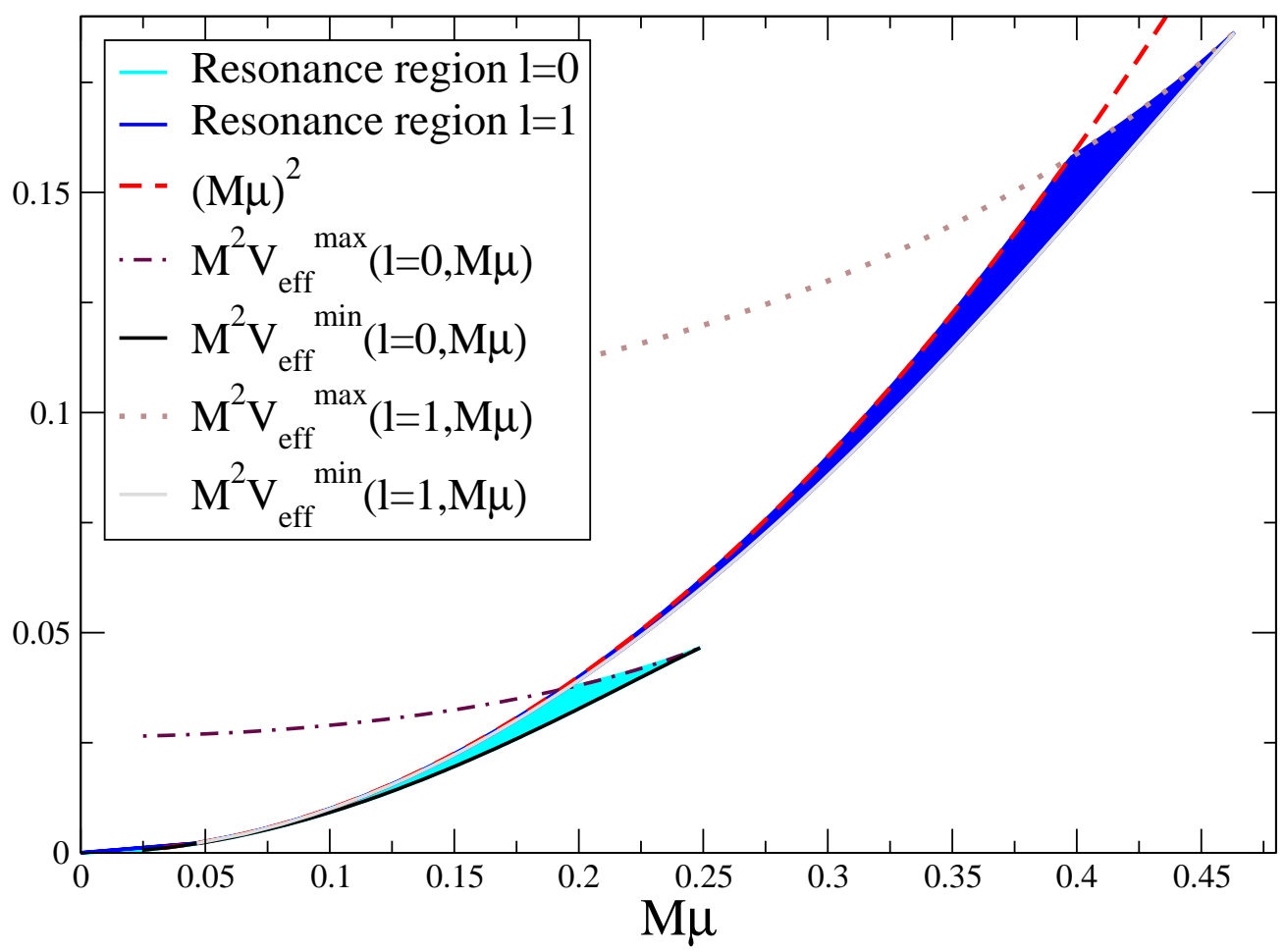

FIG. 2. Resonance band in parameter space. The cut-off in $M \mu$ is reached for values so large that condition (15) is no longer satisfied. For $\ell=0$ this condition is $M \mu<1 / 4$, while for $\ell=1$ one finds $(M \mu)^{2}<-9 / 32+\sqrt{20577} / 288 \approx 0.217$, which implies $M \mu \lesssim 0.466$.

minimum in the effective potential for $\ell=0$, but not for $\mu=0$. This is the reason why the phenomenology of the problem we are analyzing here is quite different to that encountered in the usual Regge-Wheeler equation.

Having found under which conditions there is a potential well, we now turn our attention to the existence of resonant modes. In order to have modes that are "almost trapped" in the potential well it is clear that one must ask for $0<\omega^{2}<\mu^{2}$. This will guarantee that the solution for the scalar field decays exponentially at spatial infinity and is "localized" close to the black hole, but of course the scalar field can still "escape" towards the black hole horizon. The situation we are considering is as follows: We are solving the stationary Klein-Gordon equation, so close to the horizon (where $r^{*}$ approaches $-\infty$ ) we must have a combination of incoming and outgoing modes of the same amplitude. This implies that any scalar field that "escapes" from the potential well (via "quantum tunneling") is precisely compensated by the incoming field from the horizon. The situation is clearly not physical 
since, first, we do not expect anything coming from the horizon, and second, the energy of such solutions is infinite as discussed above, but nevertheless these are the type of solutions we are considering here.

Now, as already remarked, the frequency spectrum is continuous, so we will find a solution with the properties just described for any $0<\omega^{2}<\mu^{2}$. Clearly, if we want to find solutions that are in some sense "trapped" inside the potential well, we must also ask for $\omega^{2}$ to be in the resonance band, $V_{\text {eff }}^{\min }<\omega^{2}<\min \left\{V_{\text {eff }}^{\max }, \mu^{2}\right\}$. The question now is what distinguishes the set of discrete resonant solutions from all other solutions in the continuous spectrum of the resonance band. There are several ways in which one can look for such resonant states, but here we have settled on a practical "intuitive" approach: Resonant states will correspond to those that "leak out" of the potential well very slowly, or in other words, they will correspond to those special solutions for which the ratio between the amplitude outside the potential well and the amplitude inside is a minimum.

In order to have a clear picture of what we are talking about here, let us consider the particular case $\ell=1, M \mu=0.3$, which clearly satisfies the conditions for a potential well to exist. Figure 1 shows the effective potential $M^{2} V_{\text {eff }}$ in that case. Notice that there is a clear minimum at $r^{*} / M \approx 25$ for which $M^{2} V_{\mathrm{eff}}^{\min } \approx 0.085$, while the maximum is at $r^{*} / M \approx 2.2$ with $M^{2} V_{\mathrm{eff}}^{\max } \approx 0.13$.

We can now solve the Schrödinger equation (9) numerically for an arbitrary value of $0<\omega^{2}<\mu^{2}$. The details for the numerical solver are not particularly important, it is sufficient to say that the solver starts at a large value of $r^{*} / M$, for which $V_{\text {eff }} \approx \mu^{2}$, and where one can approximate the solution with a decaying exponential. One then solves the equation numerically from right to left.

Figure 3 shows two solutions for particular values of $M \omega$. The first plot corresponds to the solution for $M \omega=0.295$. Notice that for $r^{*} / M<0$ the solution is oscillatory as expected, and that it penetrates only slightly into the potential well: The amplitude for $r^{*} / M<0$ is much larger than the amplitude inside the well. This behavior is quite generic. The second plot, on the other hand, correspond to the solution for $M \omega=0.29619$. Notice that now the situation is quite the opposite: The amplitude inside the well is much larger than the amplitude for $r^{*} / M<0$ (both solutions have been scaled so that the maximum overall amplitude is equal to 1 ). This means that the value $M \omega=0.29619$ is close to a resonant frequency; in fact it is close to the very first resonance, the one with no nodes 


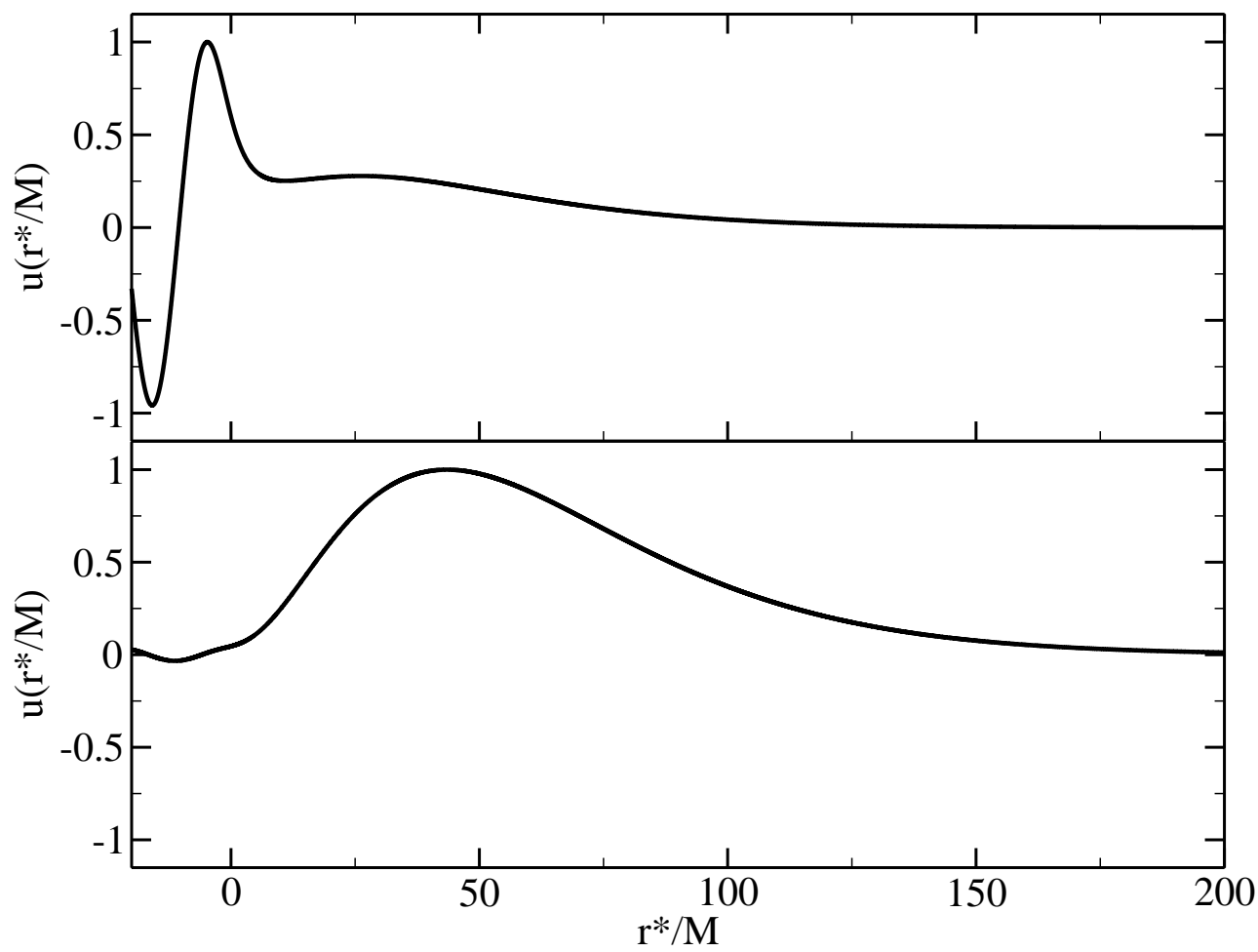

FIG. 3. Numerical solutions of the Schrödinger equation (9) for different values of $M \omega$. The upper panel corresponds to $M \omega=0.295$, while the lower panel corresponds to $M \omega=0.29619$. Both solutions have been scaled so that the maximum overall amplitude is equal to 1.

inside the potential well.

In order to locate the different resonant frequencies one can find the solution for all frequencies in the interval $V_{\text {eff }}^{\min }<\omega^{2}<\mu^{2}$, and simply plot the ratio between the amplitude $A_{\text {out }}$ of the solution for $r^{*} / M<0$, and the amplitude $A_{\text {in }}$ for the solution inside the potential well. Figure 4 is just such a plot for the case we are considering here. Notice that the ratio $A_{\text {out }} / A_{\text {in }}$ drops to very small values at a set of sharply defined discrete frequencies. Each subsequent resonant frequency can be easily shown (by just plotting them) to correspond to solutions with more nodes inside the potential well. As the value of $\omega$ approaches the parameter $\mu$ the resonant frequencies become closer to each other, and in fact there seem to be an infinite number of them. Care must be taken when finding the numerical solution, since higher resolution is required as $\omega$ approaches $\mu$ (both in $r^{*} / M$ and in $\omega$ ), while at the same time one needs to consider much larger values of $r^{*} / M$ since the solutions become so much wider (specific examples of such resonant solutions will be considered in the following 


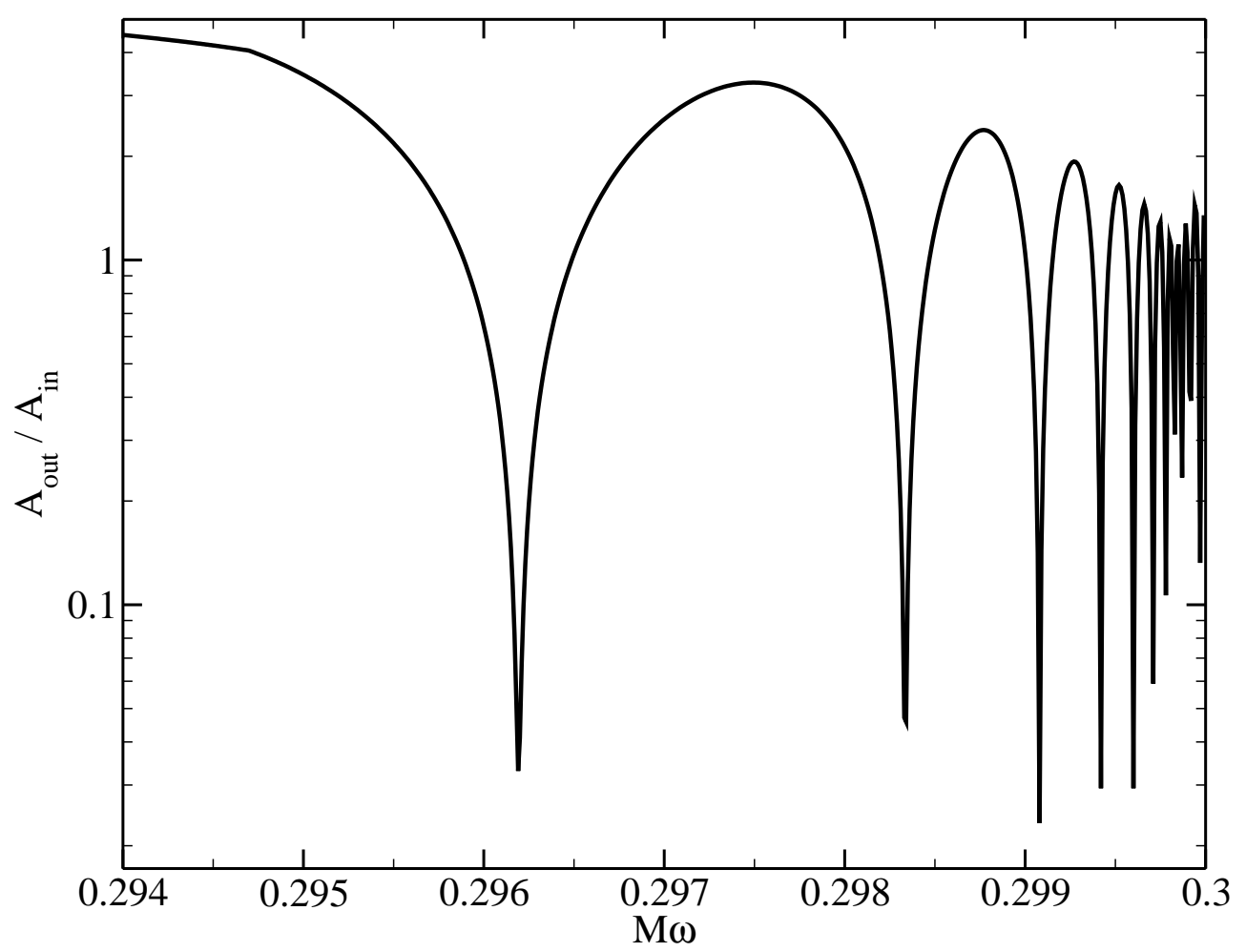

FIG. 4. Resonant frequencies. We show a log plot of the ratio $A_{\text {out }} / A_{\text {in }}$ between the amplitude $A_{\text {out }}$ of the solution for $r^{*} / M<0$, and the amplitude $A_{\text {in }}$ for the solution inside the potential well $r^{*} / M>10$.

sections).

\section{LONG LASTING CONFIGURATIONS. NUMERICAL STUDY}

According to the arguments given in Section 1 above, we can not have stationary, localized, physical scalar field configurations surrounding a Schwarzschild black hole: The stationary solutions, even the resonant ones, have a divergent energy due to their oscillatory behavior close to the horizon. 4 However, one can always construct physical configurations that are arbitrarily close to the stationary solutions and that can survive the black hole for arbitrarily long times: Simply choose any (non-physical) stationary solution that decays at spatial infinity (i.e. $0<\omega^{2}<\mu^{2}$ ), and set it to zero by hand in the interval $r \in(2 M, 2 M+\epsilon)$, for some small parameter $\epsilon>0$ with dimensions of length. The resulting

\footnotetext{
${ }^{4}$ Moreover, such solutions are not compatible with the test field limit approximation.
} 
configuration can be seen as a combination of the stationary solution plus a perturbation around the horizon, and it will be stationary for the domain of dependence of $[2 M+\epsilon, \infty)$.

For a given value of $\epsilon$, and some $\Delta>\epsilon$, it will take a time of order $t_{\epsilon}(2 M+\Delta)=$ $2 M \ln (\Delta / \epsilon)+(\Delta-\epsilon)$ for the perturbation to reach the radial coordinate $r=2 M+\Delta$. That is, the standard flat space-time result $\Delta-\epsilon$, plus an extra term coming from the non-trivial space-time curvature, $2 M \ln (\Delta / \epsilon)$. In the limit $\epsilon \rightarrow 0$ that time can be made arbitrarily large. Since the energy density diverges as $r \rightarrow 2 M$, one could naively think that we can not make $\epsilon$ arbitrarily small if we want to remain within the test field approximation, but notice that, since the Klein-Gordon equation is linear in $\phi$, for any given value of $\epsilon$ one can always reduce the amplitude of the scalar field arbitrarily. But of course, that would yield scalar field distributions in which most of the energy is concentrated very close to the horizon.

If we take $2 M+\Delta$ to be of the order of the size of a given scalar field distribution surrounding a Schwarzschild black hole, one would expect that $t_{\epsilon}(2 M+\Delta)$ could give us some insight into the lifetime of that particular configuration. 5 It is worth noticing, however, that $t_{\epsilon}$ works only as a lower bound, and some scalar field distributions could very well last much longer than that. In fact, we will show that initial data can be constructed in a particular way that produces long lasting, quasi-stationary scalar field distributions in a compact region outside the black hole. These initial data correspond to the resonant states, and we will devote most of the remaining of this paper to study them, although we will also consider a few other configurations for comparison. We will come back to the study of the characteristic time-scales associated to these configurations in Section IIC2.

\section{A. Initial Data}

We will now construct pseudo-stationary configurations by truncating the stationary solutions as explained in the previous section. These configurations will then be used as initial data to be evolved numerically so that we can study the energy decay in a compact region outside the black hole. As already mentioned, the spectrum of stationary solutions is continuous, however we will show that pseudo-stationary initial data constructed from a particular discrete subset of stationary solutions, that of the resonant states, yields long

${ }^{5}$ In fact, for the configurations we will be considering here (see Table凹), this lifetime will not be particularly sensitive to the parameter $\epsilon$. If the width of the scalar field configuration is of order $10^{3} \mathrm{M}$, then one needs to take $\epsilon \lesssim 10^{-11}$ for the gravitational term $2 M \ln (R / \epsilon)$ to be relevant in the expression for $t_{\epsilon}$. 
lasting configurations.

As described above, we construct initial configurations that correspond to stationary solutions in the domain of dependence of the region $r>2 M+\epsilon$, with $\epsilon \lesssim 0.05 M$. To do so, we integrate Eq. (8) numerically in the region $\left[R_{\text {in }}=2 M+\epsilon, R_{\text {out }}\right]$ using a shooting algorithm (see e.g. [51]). As boundary condition we impose at $r=R_{\text {out }}$ the relation $u^{\prime}=-|k| u$ with the constant $k$ defined in Eq. (12) to ensure that $u(r)$ falls exponentially at large $r$ (see also [46] for more details). For the left boundary condition we just impose $u\left(R_{\text {in }}\right)=1$, since the equation is homogeneous so $u(r)$ can be rescaled afterwards. Finally, $u(r)$ is simply set to 0 for $r<R_{\text {in }}$. This introduces a discontinuity in the scalar field configuration, but the discontinuous jump is very small compared to the maximum absolute value of the scalar field and does not seem to introduce any numerical artifacts.

Leaving the value of $\epsilon$ aside, these initial configurations are characterized by the parameters $\ell$ and $M \mu$, which parametrize the effective potential $M^{2} V_{\text {eff }}\left(r^{*} / M\right)$, together with $M \omega$ (see Eqs. (9) and (10) above). Here we will restrict ourselves to the region $0<\omega^{2}<\mu^{2}$. The solutions with $\omega^{2}>\mu^{2}$ are not localized close to the black hole and they will not be interesting for our purposes here. For the subspace with $0<\omega^{2}<\mu^{2}$ one can distinguish the following cases:

1. Configurations constructed from stationary solutions in the resonance band, i.e. with $V_{\text {eff }}^{\min }<\omega^{2}<\min \left\{\mathrm{V}_{\text {eff }}^{\max }, \mu^{2}\right\}$.

(a) Pseudo-stationary configurations constructed from resonant stationary solutions. We will call these pseudo-resonant states.

(b) Pseudo-stationary configurations constructed from non-resonant stationary solutions. We will call these non-resonant states.

2. Configurations constructed from stationary solutions outside the resonance band.

From now on we will also refer to these configurations as type 1a, type $1 \mathrm{~b}$ and type 2 , respectively. Here we are assuming that the effective potential does have a local minimum for some value of $r^{*}$, i.e. Eq. (15) is fulfilled. If that is not the case, all the configurations will be of type 2 .

Examples of type $1 \mathrm{a}$ and type 2 configurations are shown in Figure 5 , where we plot the effective potential, together with two eigenvalues $(M \omega)^{2}$ (top panel), and the radial 
energy density of the corresponding eigenfunctions (bottom panel). The Figure is shown in Eddington-Finkelstein coordinates, more appropriate for the numerical evolution (see Section [IIB). For this particular case we have chosen $\ell=1$ and $M \mu=0.3$, so that Eq. (15) is fulfilled and a potential well is present (that case was also considered in Section IIC). One would expect that such solutions will have an oscillatory behavior in regions where $V_{\text {eff }}<\omega^{2}$, with most of the scalar field energy contained in those regions; and with a rapid decay outside. To help guide the eye, we added vertical lines to the plots at the locations where $V_{\text {eff }}=\omega^{2}$ (classical turning points). We can see that most of the scalar field is located inside the classically permitted regions $\left(V_{\text {eff }}<\omega^{2}\right)$. In particular, the type 2 configuration, for which $\omega^{2}<V_{\mathrm{eff}}^{\min }$, is located mostly to the left of the centrifugal barrier. A type 1b configuration has not been shown here, but we can look at these configurations as somehow intermediate between type 1 a and type 2 .

We will now concentrate on type 1a configurations which, as we will see in Section IIIB, are the ones that show long lasting scalar field distributions at a compact region outside the black hole. We construct pseudo-resonant initial data configurations for different values of $\ell$ and $M \mu$, and for each pair of these parameters we study pseudo-resonant states up to the fifth mode.

Table I summarizes some properties of the pseudo-resonant state initial data we have studied here. In what follows, $R_{99}$ is defined as the radius of a sphere containing $99 \%$ of the scalar field energy $E$ at $t=0$, and gives a rough idea of the "size" of each configuration. Note that $R_{99}$ increases as $M \mu$ decreases and $n$ increases. Some of these configurations are illustrated in Figures [6 and 7. Figure 6] shows the energy density distributions $\rho_{E}$ for the first five pseudo-resonant modes $n=(1,2,3,4,5)$ for $\ell=1$ and $M \mu=0.2$, while Figure 7 shows the first mode $n=1$ pseudo-resonant configuration for different values of $M \mu$.

Note that the eigenvalues $w_{n}^{2}$ of the resonant modes accumulate at $w^{2}=\mu^{2}$ from below. It seems likely that there is in fact an infinite number of resonant modes $n$, although we have not investigated in any detail if this is indeed the case. 6 The possible existence of modes with arbitrary $n$ would be interesting, since the energy distribution seems to spread further away from the black hole for larger $n$, implying that arbitrarily wide resonant modes might be constructed.

\footnotetext{
${ }^{6}$ Note that in the case of bound states in quantum mechanics, effective potentials with an asymptotic behavior for $r \rightarrow \infty$ like the one in Eq. (10) usually have an infinite number of bound states.
} 


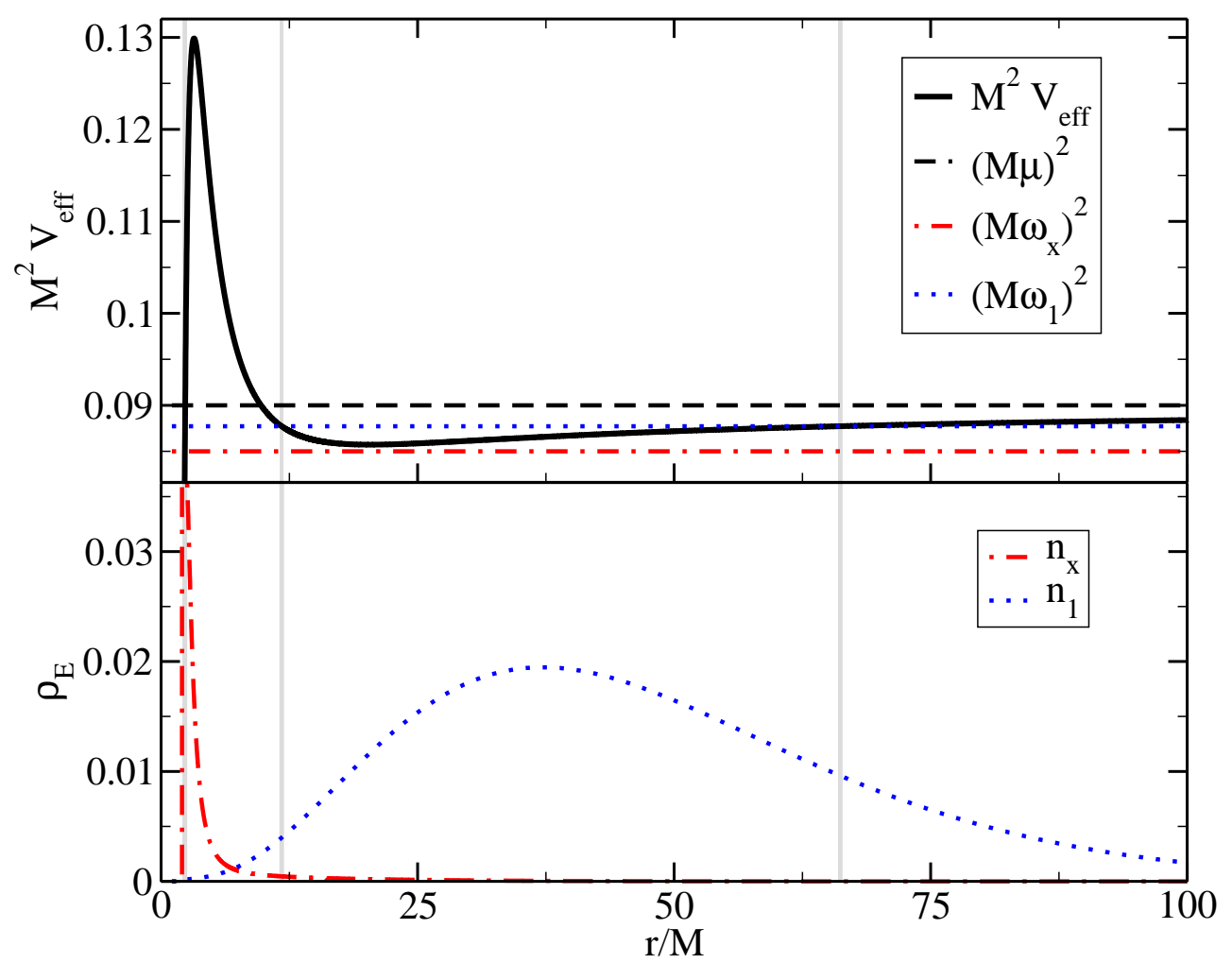

FIG. 5. Top panel: Effective potential $V_{\text {eff }}(r)$ for $\ell=1$ and $M \mu=0.3$; and $\left(M \omega_{i}\right)^{2}$ for each of the solutions shown in the bottom panel. Bottom panel: Radial energy density $\rho_{E}(r)$ for the first $\left(n_{1}\right)$ pseudo-resonant mode (type1a) and a solution (labeled $n_{x}$ ) with eigenvalue (labeled $w_{x}^{2}$ ) below the local minimum of $V_{\text {eff }}$ (type 2). An arbitrary rescaling was chosen for each $\rho_{E}$ in this plot in order to improve visualization. The vertical lines intersect the roots of $V_{\text {eff }}=w^{2}$, a total of three for the pseudo-resonant mode $\left(n_{1}\right)$, and only one for the non-resonant mode (note that the root of the non-resonant mode is very close to the first root of the resonant mode, so the two corresponding vertical lines are indistinguishable in the figure).

\section{B. Numerical Evolution}

Having constructed a large set of initial data, we now study some properties of their evolution. The numerical evolution is performed in penetrating coordinates, hence covering a region that reaches inside the event horizon and avoiding the need to provide boundary conditions there. We use ingoing Eddington-Finkelstein coordinates $(\bar{t}, r, \theta, \varphi)$, with $\bar{t}:=t-2 M \ln (1-N(r))$, and $N(r)$ defined in (2) . In these coordinates the spacetime line- 
TABLE I. For the case $\ell=1$, and different combination of parameters $M \mu$ and $n$ we show: (i) The "width" of the initial energy distribution $R_{99}$ and (ii) The eigenvalue $(M \omega)^{2}$. Notice that $R_{99} / M$ is given with three significant figure accuracy, while $(M \omega)^{2}$ is given with an accuracy up the last significant figure shown. All values shown correspond to pseudo-resonant (type 1a) states.

\begin{tabular}{cccccc}
\hline \hline$n$ & $(M \mu)^{2} \rightarrow$ & $(0.2)^{2}=0.04$ & $(0.25)^{2}=0.0625$ & $(0.3)^{2}=0.09$ & $(0.35)^{2}=0.1225$ \\
\hline \hline 1 & $R_{99} / M$ & 279 & 173 & 116 & 79.7 \\
& $(M \omega)^{2}$ & 0.03958216 & 0.061450 & 0.08773 & 0.1180 \\
\hline 2 & $R_{99} / M$ & 589 & 369 & 251 & 170 \\
& $(M \omega)^{2}$ & 0.0398152 & 0.062037 & 0.089004 & 0.1205 \\
\hline 3 & $R_{99} / M$ & 1000 & 629 & 421 & 301 \\
& $(M \omega)^{2}$ & 0.03989669 & 0.0622423 & 0.089449 & 0.1214 \\
\hline 4 & $R_{99} / M$ & 1520 & 957 & 646 & 459 \\
& $(M \omega)^{2}$ & 0.0399342 & 0.0623364 & 0.089652 & 0.12183 \\
\hline 5 & $R_{99} / M$ & 2140 & 1350 & 917 & 651 \\
\hline \hline & $(M \omega)^{2}$ & 0.0399545 & 0.0623871 & 0.089761 & 0.12204 \\
\hline \hline
\end{tabular}

element takes the form

$$
d s^{2}=-\left(1-\frac{2 M}{r}\right) d \vec{t}^{2}+\frac{4 M}{r} d \bar{t} d r+\left(1+\frac{2 M}{r}\right) d r^{2}+r^{2} d \Omega^{2} .
$$

This coordinate system covers a region that includes the black hole interior $r \in(0, \infty)$, and is regular at the horizon. Notice that for the purposes of our simulations the Schwarzschild and Eddington-Finkelstein times can be considered equivalent since at a fixed radial coordinate $r$ one can easily see that $\Delta t=\Delta \bar{t}$. Because of this, and in order to simplify the notation, from now on we will drop the bar from the Eddington-Finkelstein time and will refer to it simply as $t$.

In order to find numerical solutions to the Klein-Gordon equation, we define a set of first order derivatives, and obtain a system of evolution equations of the form $\partial_{t} \vec{\Psi}+B \partial_{r} \vec{\Psi}=S$, with $B$ a symmetric matrix and $\vec{\Psi}$ the vector formed from first derivatives in space and time of $\psi_{l m}$. See references [46, 52] for details.

The evolution equations are solved numerically using second order finite differences in space, and evolving in time using a method of lines with a third order Runge-Kutta inte- 


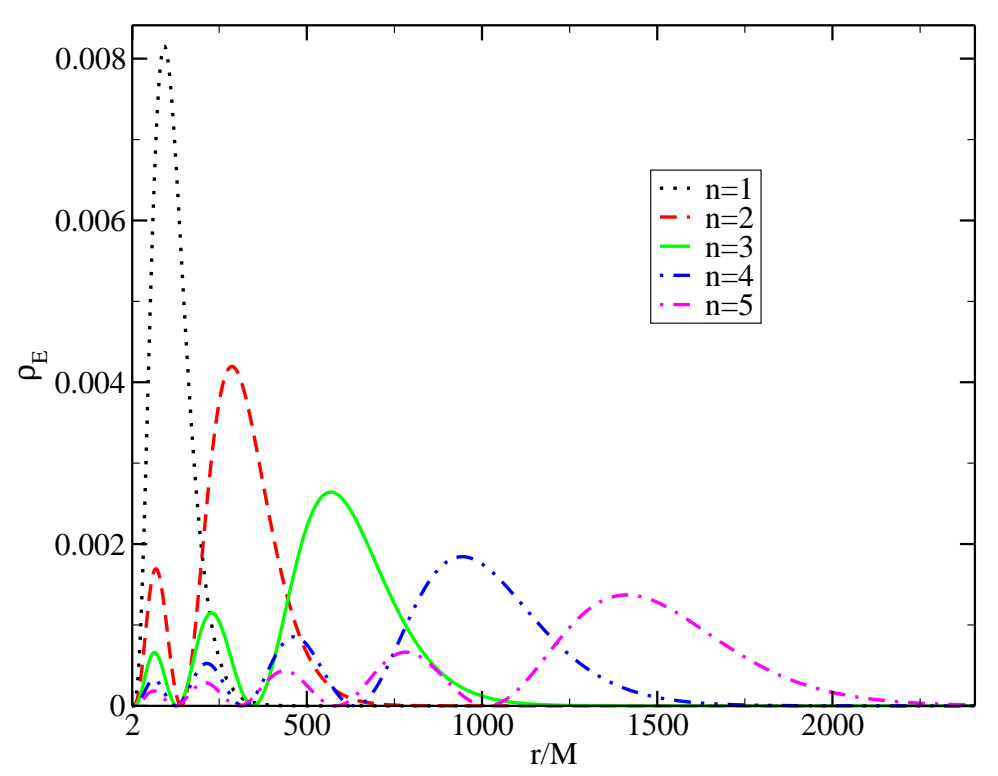

FIG. 6. Radial energy density $\rho_{E}$ at $t=0$ for different pseudo-resonant (type 1a) modes $n=(1,2,3,4,5)$, for the case $\ell=1$ and $M \mu=0.2$. Notice that the solution has been normalized so that we always have $E_{l m}=1$.

grator. Since we are using horizon penetrating coordinates we set the left boundary $r_{\min }$ inside the event horizon, leaving the boundary conditions "free" at that point (by using one-sided spatial differences), since that region is causally disconnected from the exterior. On the other hand, the right boundary $r_{\max }$ is set far away and all incoming modes are set to zero there. The code passed the standard convergence and residual evaluation tests. Furthermore, two independent codes have been used in this work, and comparisons give identical results up to truncation error.

The resolution used varies depending on the initial data. Typically, the region containing most of the scalar field (i.e. from $r_{\min }$ to about $R_{99}$ ) is covered with at least 2,000 , and often as much as 32,000 , grid points. Then, the domain is chosen so that $r_{\max } \approx 4 R_{99}$ or larger. Note that such high resolutions are needed in some cases in order to calculate the parameter $s$, defined below, with a relative error smaller than one. This is mainly due to its proximity to zero. 


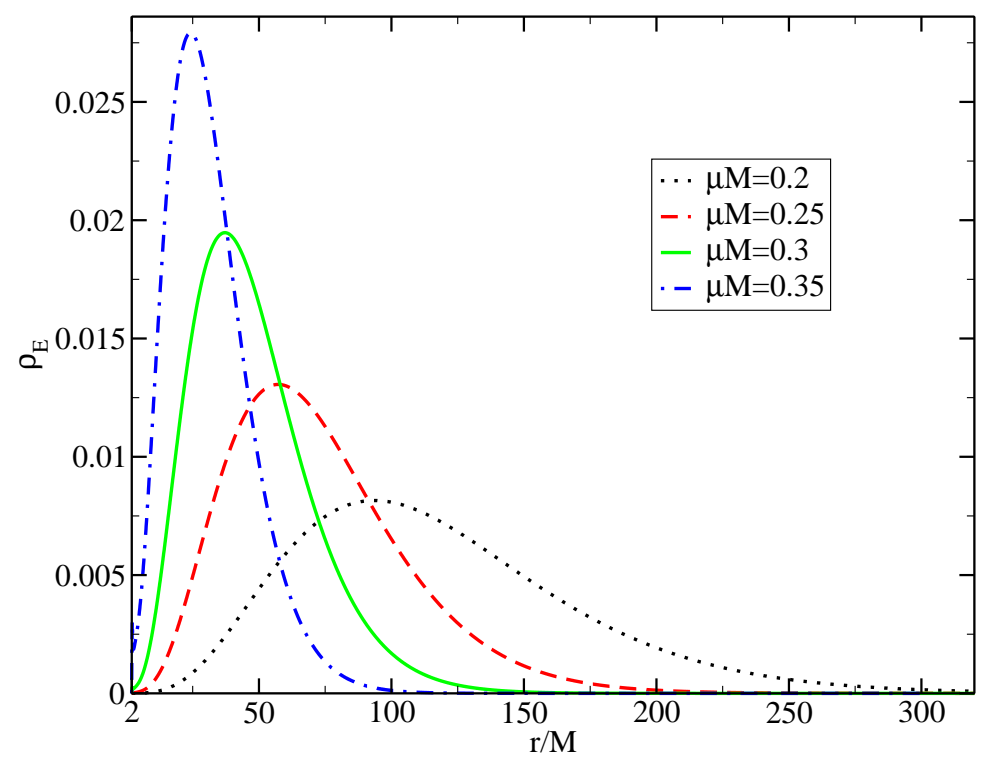

FIG. 7. Radial energy density $\rho_{E}$ for $\ell=1$ and the first pseudo-resonant (type 1al mode $n=1$, for configurations with different values of $M \mu$. The solution has been normalized so that $E_{l m}=1$.

\section{Results}

We study the evolution of the scalar field $\psi_{l m}(t, r)$ for different initial data configurations. Although our attention is mainly focused on the evolution of the long lasting pseudo-resonant states, we also consider other configurations for comparison. We start by evaluating total energy loss and studying some spectral characteristics of the different configurations by means of a time Fourier analysis, and finish with more explicit considerations about how long can such configurations last.

\section{Energy Decay and Spectral Analysis}

In order to evaluate how long a scalar field configuration remains confined in a compact region we evaluate the conserved energy $E_{l m}$, defined in equation (6) , but we replace the upper limit of integration with a finite value $R>R_{99}$, with typical values of $R$ between $2 R_{99}$ and $10 R_{99}$.

We begin by studying the evolution of the pseudo-resonant initial data (type 1a) presented in table 1, Figures 8 and 9 show the evolution of the integrated scalar field energy, $E(t)$ (we 
will drop the $\ell m$ sub-indexes from now on), for some of the parameters $M \mu$ and $M \omega_{n}$ studied. Notice that the energy is plotted on a logarithmic scale, and shows a slow exponential decay of the form

$$
E(t)=E_{0} \exp (-s t / M)
$$

with $s$ constant, except for some very small oscillations that remain during the whole evolution (these oscillations are noticeable in the overlay frames in the figures). Given the exponential decay that dominates the overall behavior, we can perform a linear fit of $\ln \left(E / E_{0}\right)$ as a function of $t / M$ to calculate the parameter $s$. These values are shown in Table $\prod$ below. In all the cases considered here no energy is seen to escape to infinity, but it rather falls into the black hole. In Section IIIC2 below we will evaluate explicitly the characteristic times for these configurations. The results shown on the table correspond to the evolution of pseudo-stationary initial data constructed from the discrete spectrum of resonant stationary states (type 1a).

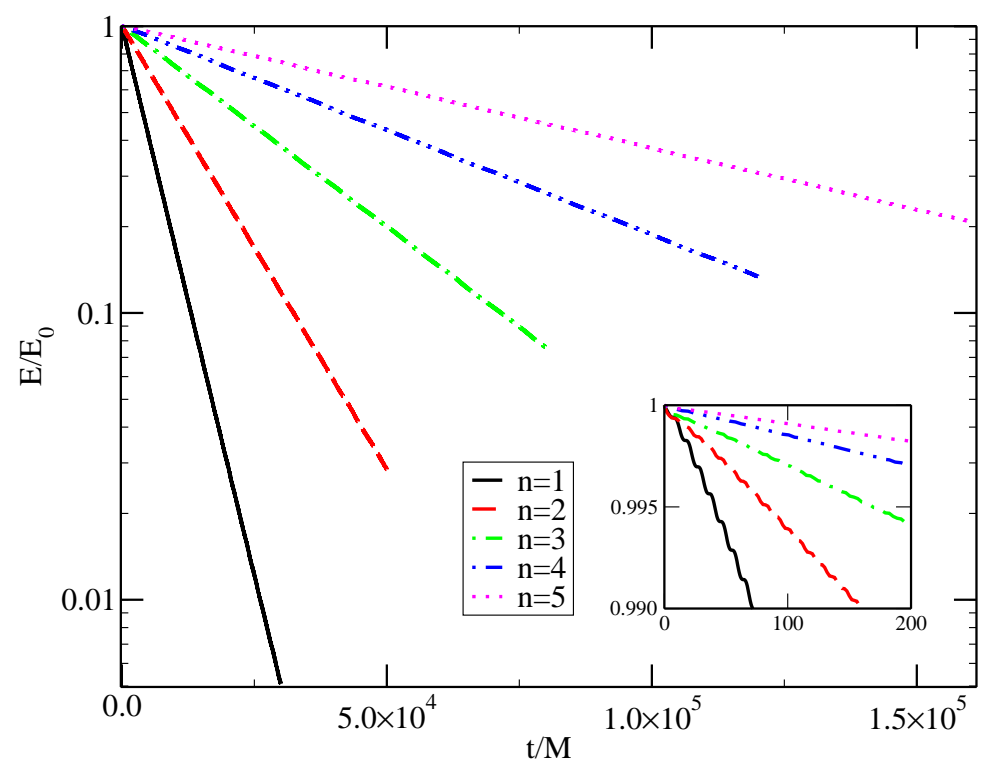

FIG. 8. Energy of the scalar field vs. time for the evolution of pseudo-resonant (type 1ad) initial data with $\ell=1, M \mu=0.35$ and modes $n=(1,2,3,4,5)$. As can be clearly seen some evolutions last longer than others, this is because we chose to run each case for a time length approximately proportional to the size of the scalar field distribution, $R_{99}$.

We have also evolved a couple of type $1 \mathrm{~b}$ configurations, that is, non-resonant pseudostationary states. Such non-resonant states do not evolve in a quasi-stationary fashion, 


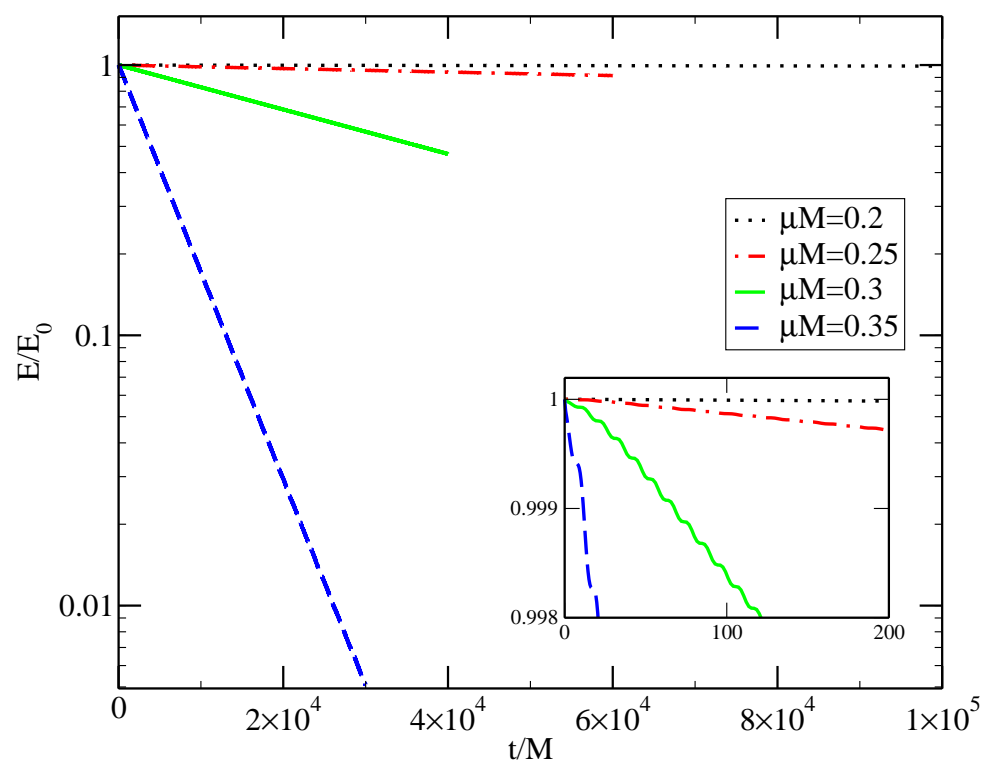

FIG. 9. Energy of the scalar field vs. time for the evolution of the initial data corresponding to the first $(n=1)$ pseudo-resonant (type 1a mode with $\ell=1$, and $M \mu=0.2,0.25,0.3$ and 0.35 .

TABLE II. For the case with $\ell=1$, and each combination of parameters $M \mu$ and $n$, we show the slope $s$ of a linear fit of $\ln \left(E(t) / E_{0}\right)$, as described in the text. The error in the slope is estimated either as one half the difference of the values obtained with the two higher resolutions used in each case, or as the error obtained from the linear regression, whichever is larger.

\begin{tabular}{l|rrrr}
\hline \hline$n$ & \multicolumn{4}{|c}{$(M \mu)^{2}$} \\
& $(0.20)^{2}=0.04$ & $(0.25)^{2}=0.0625$ & $(0.30)^{2}=0.09$ & $(0.35)^{2}=0.1225$ \\
\hline 1 & $(8.14 \pm 0.05) \times 10^{-8}$ & $(1.49 \pm 0.01) \times 10^{-6}$ & $(1.857 \pm 0.002) \times 10^{-5}$ & $(1.657 \pm 0.001) \times 10^{-4}$ \\
2 & $(2.96 \pm 0.03) \times 10^{-8}$ & $(5.53 \times 0.02) \times 10^{-7}$ & $(7.20 \pm 0.05) \times 10^{-6}$ & $(6.477 \pm 0.005) \times 10^{-5}$ \\
3 & $(1.3 \pm 0.1) \times 10^{-8}$ & $(2.479 \pm 0.005) \times 10^{-7}$ & $(3.24 \pm 0.02) \times 10^{-6}$ & $(2.904 \pm 0.007) \times 10^{-5}$ \\
4 & $(7 \pm 2) \times 10^{-9}$ & $(1.30 \pm 0.01) \times 10^{-7}$ & $(1.690 \pm 0.006) \times 10^{-6}$ & $(1.506 \pm 0.002) \times 10^{-5}$ \\
5 & $(5 \pm 4) \times 10^{-9}$ & $(7.6 \pm 0.2) \times 10^{-8}$ & $(9.83 \pm 0.01) \times 10^{-7}$ & $(8.61 \pm 0.07) \times 10^{-6}$ \\
\hline \hline
\end{tabular}

instead they very quickly lose a significant portion of their energy, some falls into the black hole while some is radiated toward infinity. It is only after quite some time has passed that they reach a quasi-stationary state, and with much less than their original energy. Once they are in the quasi-stationary regime, the energy falloff is very similar to that of the pseudo-resonant states. Each non-resonant state seems to evolve, at least after some time, 
as a combination of pseudo-resonant modes.

To understand this behavior better we perform a spectral analysis. We calculate the discrete Fourier transform in time of the numerical scalar field evolutions. More precisely, we calculate the magnitude of the discrete Fourier transform in time of the field $\psi(t, r)$ at a fixed spatial point (or points) $r=r_{j}$, located approximately at a local extremum of $\psi(t=0, r)$, and pay attention to possible differences derived from using different sample points $r_{j}$. The explicit expression is

$$
F[\psi(t)](f):=\left|A \sum_{p} \psi\left(t_{p}, r_{j}\right) \exp \left(-2 \pi i f t_{p}\right)\right|,
$$

where $A$ is a normalization constant and $t_{p}$ are the discrete time values.

The Fourier transform of a (type 1a) pseudo-resonant state is shown in Figure 10, and that of two (type 1b) non-resonant ones is shown in Figures 11 and 12. For this example we have chosen to show an $n=2$ pseudo-resonant state and two non-resonant states with frequencies $\omega_{x}$ between the first and second pseudo-resonant modes $\left(\omega_{1}<\omega_{x}<\omega_{2}\right)$. These two frequencies have been denoted with an $x$ in the overlay figures, while numbers from 1 to 5 denote the first five resonant frequencies. All cases correspond to $\ell=1$ and $M \mu=0.3$.

Figures 10 and 11 show the Fourier transform at a single sample point $r_{1}$. While the results shown in these two figures are almost independent of $r_{1}$, for the case considered in Figure 12 there were some small differences depending on whether the sample point $r_{j}$ was chosen close to the first or the second local extremum, so we show both results in that case. Note however that the two curves in Figure 12 are qualitatively identical, and the peaks are located at exactly the same frequencies, the conclusions we will make from this figure are then independent of the sample point chosen.

Having mentioned a few technical details about the spectral analysis, we now make some observations. Consider first the spectrum of the pseudo-resonant mode with frequency $\omega_{2}$ shown in Figure 10. As might be expected for this configuration, we see a single and well defined peak at exactly $\omega_{2}$. Note however that this result is not so obvious a priori, since we are actually evolving a truncated version of the actual stationary state. Consider next the non-resonant configurations, Figures 11 and 12, with frequencies $\omega_{x}$. We note that no peaks are present in the spectrum at the frequency $\omega_{x}$, instead one can see clear peaks at different resonant frequencies $\omega_{n}$. Note also that, although not strictly true for all cases, the peaks tend to be higher the closer they are to $\omega_{x}$. It would seem that, after some 


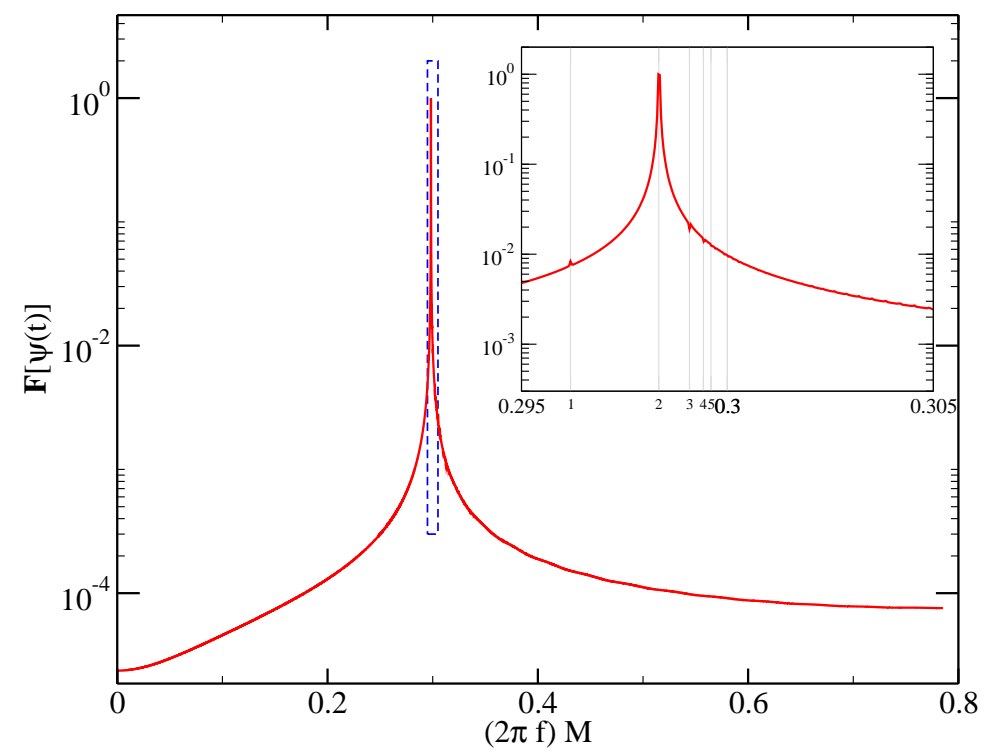

FIG. 10. Discrete Fourier transform in time vs. frequency for the evolution of type 1a data with $M \mu=0.3, \ell=1$, and frequency $w_{2}$ (corresponding to the second pseudo-resonant mode). The overlay figure shows in more detail the region contained in the dashed line rectangle. Numbers going from 1 to 5 in the overlay figure denote the first five resonant frequencies.

transient, these non-resonant states evolve as a combination of pseudo-resonant states. In that sense, it seems that the pseudo-resonant states could play a similar role to that played by the quasi-normal modes in the study of perturbed black holes. This point should be investigated further, but it is outside the scope of the present paper.

The Fourier transforms were calculated integrating over a time interval that goes from $t=0$ to $t \approx 10^{5} \mathrm{M}$. Such a large interval is necessary to ensure that the final result has enough frequency resolution to distinguish between the different resonant frequencies $\omega_{n}$, which are very close to each other. This fact also implies that we cannot integrate over shorter intervals in order to evaluate possible time variations of the spectrum at the beginning of the evolution, before reaching a quasi-stationary state.

\section{Characteristic Timescales}

We now turn our attention back to the evolution of pseudo-resonant (type 1a) configurations. Based on the results outlined in table $\amalg$ we can be more explicit about how long this states can last. If the exponential regime observed is sustained for all times, a characteristic 


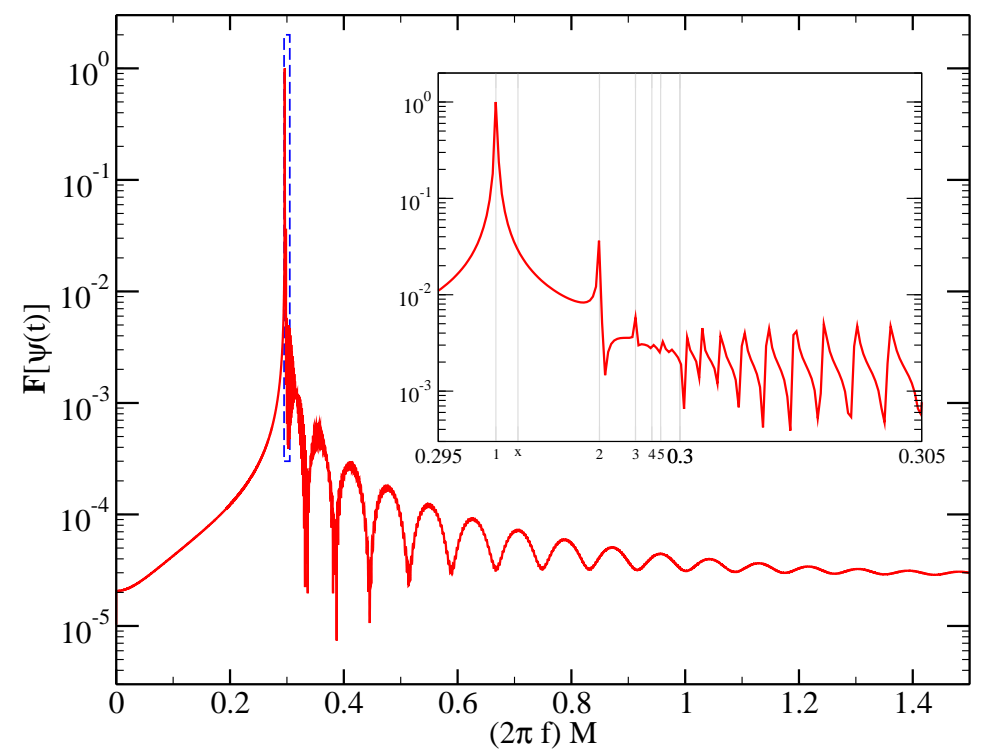

FIG. 11. Discrete Fourier transform in time for the evolution of type 1 b data with $M \mu=0.3, \ell=1$, and frequency $w_{x}=0.29664794$. The overlay figure shows in more detail the region contained in the dashed line rectangle. Numbers going from 1 to 5 in the overlay figure denote the first five resonant frequencies, while the $x$ denotes $w_{\mathrm{x}}$.

time of these configurations, their half-life time, will be given by $t_{1 / 2}:=\ln (2) M / s$, with $s$ the parameter for the exponential decay of Eq. (18). Note that in some of the cases considered we were able to evolve far beyond $t_{1 / 2}$ thus corroborating, at least for those parameters, that the exponential regime is indeed sustained (see Fig. 8).

Changing back from geometric units, and considering a black hole mass of $10^{8} M_{\odot}$, we get a characteristic time of order

$$
t_{1 / 2} \approx \frac{680}{s} \text { seconds }
$$

Replacing, for instance, the value $s=7 \times 10^{-9}$ that corresponds to $\ell=1, M \mu=0.2, n=4$ (see Table【I), we obtain $t_{1 / 2} \approx 3,000$ years. This is still a very small time when compared to cosmological time-scales, however, values of the parameter $\mu$ motivated by dark matter scalar field models correspond to $\hbar \mu \sim 10^{-24} \mathrm{eV}$ in physical units (see Introduction), or equivalently $M \mu \sim 10^{-6}$ in geometric units, and noticing how fast the parameter $s$ seems to decrease with decreasing $M \mu$ (see Table $\amalg$ ), one might expect that configurations with values of $M \mu$ motivated by dark matter scalar field models will have half-life times of cosmological scales. We were, however, unable to study cases with such small $\mu$, so the preceding argument is 


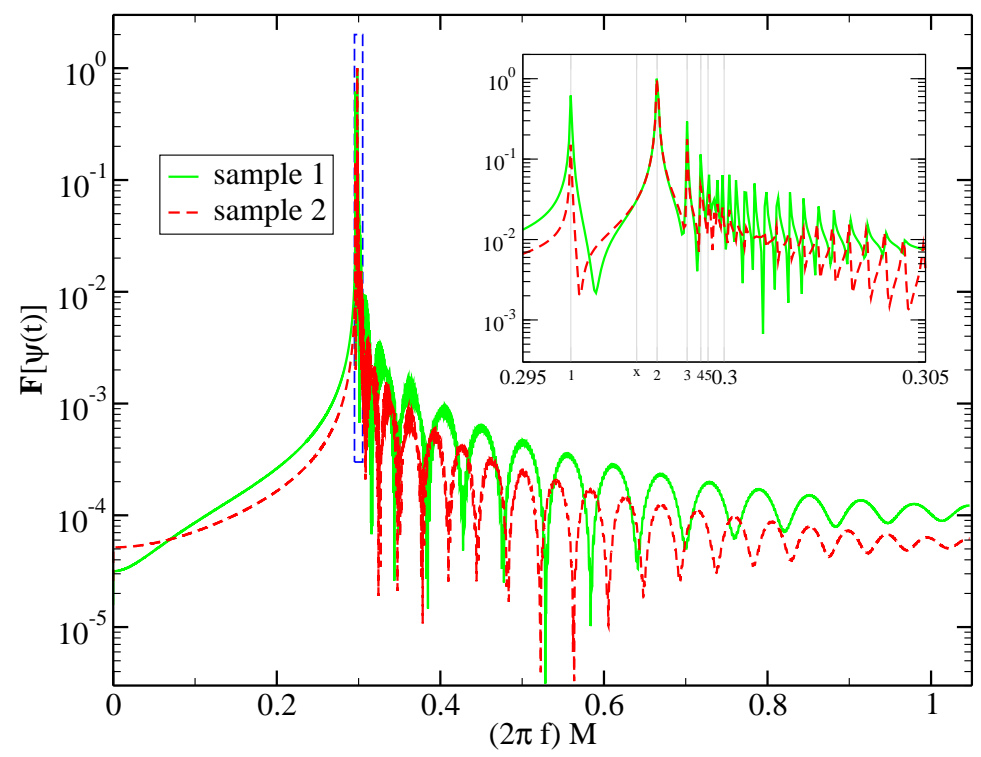

FIG. 12. Discrete Fourier transform in time for the evolution of type $1 \mathrm{~b}$ data with $M \mu=0.3, \ell=1$, and frequency $w_{x}=0.29782545$. The overlay figure shows in more detail the region contained in the dashed line rectangle. Numbers going from 1 to 5 denote the first five resonant frequencies, respectively, while the $x$ denotes $w_{\mathrm{x}}$. The solid line curve (sample 1) corresponds to a sample point located close to the first extremum, while the dashed line curve (sample 2) correspond to one close to the second extremum.

purely hypothetical.

Note that extrapolating to values of $M \mu$ of order $10^{-6}$ from the few values given in Table II which are of order $10^{-1}$, would be extremely inaccurate. If, however, we were to proceed anyway with such an extrapolation, we would obtain $s$ of about $6 \times 10^{-14}$, implying a value of $t_{1 / 2}$ of about $10^{9}$ years. Compare this value with the expected age of the universe, $10^{10}$ years. 7

Finally, for the purpose of comparison with the long lasting (1a) pseudo-resonant configurations, we will consider the evolution of configurations of type (2). In the solid-line curve of Fig. 13 we show the half-life time $t_{1 / 2}$ for both pseudo-resonant states and states outside the resonance band for the case $\ell=1$ and different values of $M \mu$. A change in the slope can be seen as soon as the critical mass $M \mu=0.466$ is reached, showing that $t_{1 / 2}$ is indeed larger for type (1a) states than for type (2) states. Here $t_{1 / 2}$ is expressed in the

\footnotetext{
7 In the parameter range studied, $s$ decreases slightly faster than an exponential with decreasing $\mu$. For the extrapolation we assume an exponential that fits the points with smaller $M \mu, M \mu=0.2$ and $M \mu=0.35$.
} 


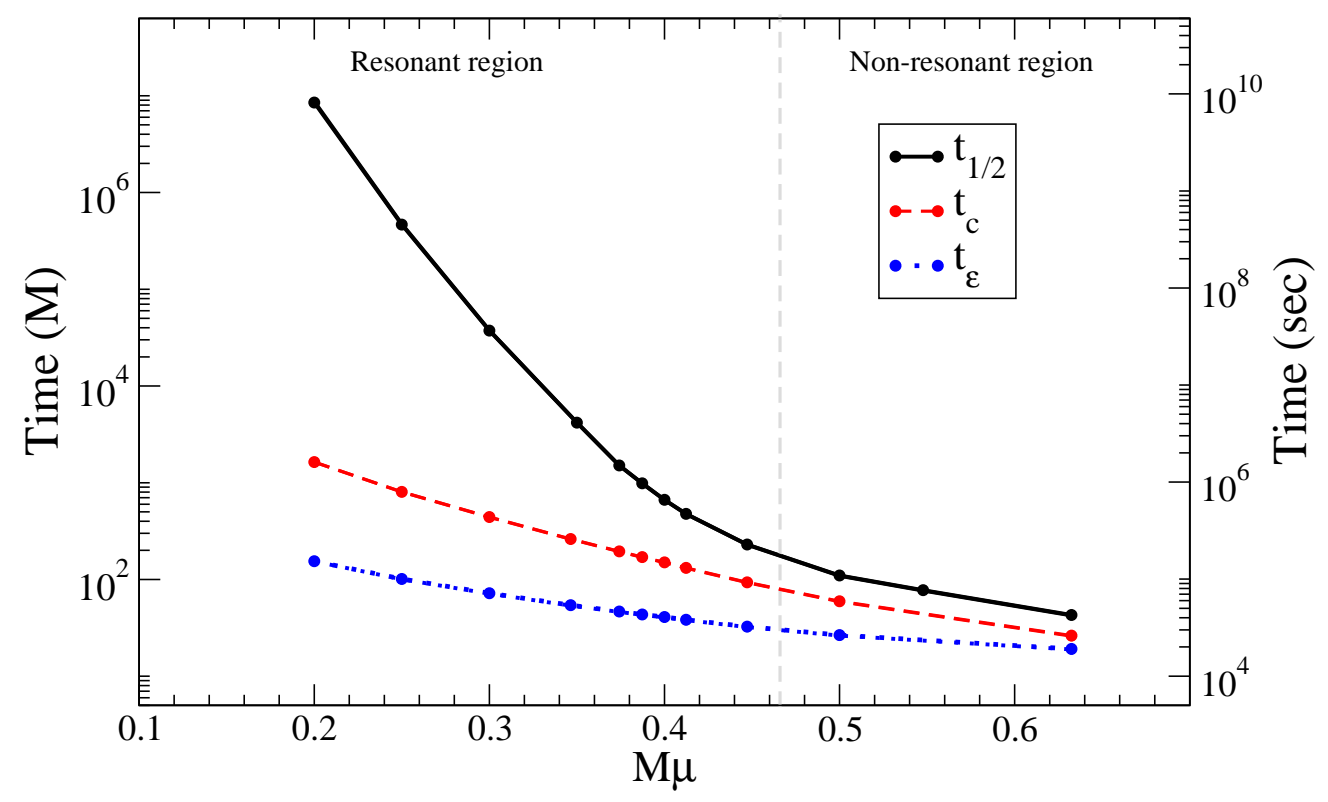

FIG. 13. Solid line: Half-life time of different scalar field configurations, $t_{1 / 2}:=\ln (2) M / s$. Dashed line: "Collapse time", defined as the time in which a pressure-less gas sphere of size $R_{99} / 2$ collapses in the gravitational field of the black hole, $t_{c}:=\sqrt{R_{99}^{3} / 8 G M}$. Dotted line: Characteristic time defined at the beginning of Section III $t_{\epsilon}\left(2 M+R_{99} / 2\right)$. The left Y-axis shows these characteristic times in units of $M$, while the right $\mathrm{Y}$-axis is expressed in seconds (assuming $M=10^{8} M_{\odot}$ ).

left vertical axis in units of $M$, while on the right vertical axis it is expressed in seconds (assuming $M=10^{8} M_{\odot}$ ).

A quick inspection of Table 1 shows that pseudo-resonant states have a $R_{99}$ larger than that of non-resonant states, i.e. they are wider. One can argue that this is the reason why pseudo-resonant states last longer. In order to show that this is not the case, we can make a comparison with some other characteristic times. For instance, we can compute the collapse time $t_{c}$, which is the time in which a pressure-less gas configuration of size $\sigma$ collapses by its own gravity. This is defined as $t_{c}:=\sqrt{\sigma^{3} / G M_{g}}$, where $M_{g}$ is the mass of the gas. In our test field approximation, $M_{g}$ will be the mass of the black hole $M_{g}=M$. In order to compare $t_{c}$ with $t_{1 / 2}$ we found appropriate to choose $\sigma$ as $R_{99} / 2$. This time is shown in the dashed-line curve of Fig. 13, As can be clearly seen in this figure, $t_{c}$ is always smaller than $t_{1 / 2}$, and for the particular case of pseudo-resonant states $t_{1 / 2}$ is orders of magnitude larger than $t_{c}$. Finally, for comparison, in the Figure we also show (dotted-line curve) the 
characteristic time $t_{\epsilon}$ defined at the beginning of Section 【II.

\section{CONCLUSIONS}

We investigated the existence of long-lasting scalar field configurations surrounding a black hole. A good motivation for this study is the possibility that super-massive black holes at galactic centers may represent a serious threat to the scalar field dark matter models. This is because such black holes may in principle swallow an hypothetical scalar field halo in cosmologically short times, which is what in general will happen with arbitrary scalar field distributions. However, we were able to find particularly long-lasting scalar field configurations around a black hole.

As a first step, we have considered a relatively simple model and looked for solutions to the Klein-Gordon equation on a Schwarzschild space-time background. Although we do find stationary solutions for the scalar field, we show that they are unphysical, in the sense that their energy density integrates to infinity in a compact region just outside the event horizon. However, we were able to find long-lasting, quasi-stationary solutions of finite energy. This is done by evolving initial data that was constructed by modifying slightly a particular subset of the (unphysical) stationary solutions. The solutions found show as an overall behavior an exponential energy decay, caused by scalar field leaking into the black hole, that in some cases can be very slow.

The stationary solutions are obtained by solving a time-independent Schrödinger-like equation with an effective potential. Hence, they can be characterized by the properties of that potential. This fact is strictly true for the stationary solutions, but interestingly we find that the (physical) quasi-stationary solutions, for which the Schrödinger-like equation no longer holds, are also characterized by properties of that same effective potential. The cases of interest are those in which the effective potential has a local minimum (a potential well). The existence of this minimum depends solely on the combination of parameters $M \mu$ and $\ell$. Although none of the possible forms of the effective potential allow for bound states, the existence of the mentioned well is enough to allow for the so-called resonant states. These states are the ones that have proven useful for constructing initial data that give rise to long-lasting quasi-stationary configurations of finite energy.

It may be objected that in order to obtain the mentioned quasi-stationary solutions 
one has to construct very particular initial data, a situation that might be very unlikely in nature. However, it seems that the crucial factor is only the existence of the potential well. We showed that even when starting with modified stationary solutions that are not resonant, after an initial abrupt energy loss, the late time behavior observed is very similar to that of the resonant quasi-stationary solutions. In fact, these solutions seem to evolve as a combination of the resonant modes. Note that the value of $\mu$ for scalar field dark matter models is expected to be given approximately by $\hbar \mu=10^{-24} \mathrm{eV}$ in physical units, or $M \mu \sim 10^{-6}$ in geometric units for a $10^{8} M_{\odot}$ black hole, which gives rise to effective potentials with a local minimum for all values of $\ell$.

When evaluating the characteristic times of our solutions we find that the longest lasting configurations last for times of the order of thousands of years. Although this is cosmologically a very short time we must note that, for technical reasons, we were only able to study cases with relatively large values of $M \mu$, of order $10^{-1}$. Noting how fast the characteristic times seem to increase with decreasing $\mu$, it would not be unreasonable to expect that configurations with $M \mu \sim 10^{-6}$ could last for cosmological time-scales. One could argue in the same lines about the "size" of the scalar field distributions: The configurations studied extend only up to a radius $R_{99} \approx 2,000 M$, but again, this number increases rapidly with decreasing $\mu$.

A few aspects of our study are still open for some improvement, and will be addressed in future works. First, a self-gravitating scalar field may be considered. Due to the extremely diluted nature of dark matter halos, the test field (Cowling) approximation is very accurate to some degree. However, given the halos large spatial extension, one expects that important differences may appear globally as a cumulative effect. Second, much smaller values of the parameter $\mu$, and much larger scalar field distributions, will be needed to do a more realistic representation of dark matter halos. The main difficulty in dealing with such configurations is handling numerically the very different scales, going from the regions close to the black hole to galactic-size scales. Mesh refinement could be used to solve this difficulty. Third, besides studying possible quasi-stationary or long-lasting configurations with an already existing black hole, it would be interesting to consider more dynamical scenarios such as the formation and/or growth of the black hole and the possibility of survival of the scalar field afterwards. We plan to address these and other issues in future works. However, the results presented here already seem to indicate that it is indeed possible for scalar field halos 
around super-massive black holes to survive for cosmological time-scales.

\section{ACKNOWLEDGMENTS}

We are grateful to the scalar field group at ICN-UNAM, for enlightening discussions. This work was supported in part by CONACyT through grants 82787 and 61173, and by DGAPA-UNAM through grant IN115311. AD, JCD and MM acknowledge DGAPA-UNAM for postdoctoral grants and $\mathrm{AB}$ and $\mathrm{JB}$ acknowledge CONACyT for postdoctoral grants. OS was also supported by grant CIC 4.19 to Universidad Michoacana de San Nicolás de Hidalgo.

[1] James E. Lidsey et al. Reconstructing the inflaton potential: An overview. Rev. Mod. Phys., 69:373-410, 1997.

[2] P. J. E. Peebles and Bharat Ratra. The cosmological constant and dark energy. Rev. Mod. Phys., 75:559-606, 2003.

[3] T. Padmanabhan. Cosmological constant: The weight of the vacuum. Phys. Rept., 380:235$320,2003$.

[4] Michael S. Turner. Coherent Scalar Field Oscillations in an Expanding Universe. Phys. Rev., D28:1243, 1983.

[5] Sang-Jin Sin. Late time cosmological phase transition and galactic halo as Bose liquid. Phys. Rev., D50:3650-3654, 1994.

[6] P. J. E. Peebles and A. Vilenkin. Noninteracting dark matter. Phys. Rev., D60:103506, 1999.

[7] P. J. E. Peebles. Fluid dark matter. 2000.

[8] Varun Sahni and Li-Min Wang. A New cosmological model of quintessence and dark matter. Phys. Rev., D62:103517, 2000.

[9] Wayne Hu, Rennan Barkana, and Andrei Gruzinov. Fuzzy Cold Dark Matter: The wave properties of ultralight particles. Phys. Rev. Lett., 85:1158-1161, 2000.

[10] Tonatiuh Matos and L. Arturo Urena-Lopez. Quintessence and scalar dark matter in the universe. Class. Quant. Grav., 17:L75-L81, 2000. 
[11] Tonatiuh Matos and L. Arturo Urena-Lopez. A further analysis of a cosmological model of quintessence and scalar dark matter. Phys. Rev., D63:063506, 2001.

[12] Ulises Nucamendi, Marcelo Salgado, and Daniel Sudarsky. An alternative approach to the galactic dark matter problem. Phys. Rev., D63:125016, 2001.

[13] Alexandre Arbey, Julien Lesgourgues, and Pierre Salati. Quintessential haloes around galaxies. Phys. Rev., D64:123528, 2001.

[14] Tonatiuh Matos, Argelia Bernal, and Dario Nunez. Flat Central Density Profiles from Scalar Field Dark Matter Halo. Rev.Mex.A.A., 44:149, 2008.

[15] Jae-Weon Lee and Sooil Lim. Minimum mass of galaxies from BEC or scalar field dark matter. JCAP, 1001:007, 2010.

[16] David J. E. Marsh and Pedro G. Ferreira. Ultra-Light Scalar Fields and the Growth of Structure in the Universe. Phys. Rev., D82:103528, 2010.

[17] Andrew P. Lundgren, Mihai Bondarescu, Ruxandra Bondarescu, and Jayashree Balakrishna. Lukewarm dark matter: Bose condensation of ultralight particles. Astrophys. J., 715:L35, 2010.

[18] Kung-Yi Su and Pisin Chen. Solving the Cusp-Core Problem with a Novel Scalar Field Dark Matter. 2010.

[19] Pau Amaro-Seoane, Juan Barranco, Argelia Bernal, and Luciano Rezzolla. Constraining scalar fields with stellar kinematics and collisional dark matter. JCAP, 1011:002, 2010.

[20] F. Briscese. Viability of complex self-interacting scalar field as dark matter. Phys. Lett., B696:315-320, 2011.

[21] T. Harko. Bose-Einstein condensation of dark matter solves the core/cusp problem. JCAP, 1105:022, 2011.

[22] Jai-chan Hwang. Roles of a coherent scalar field on the evolution of cosmic structures. Phys. Lett., B401:241-246, 1997.

[23] Wayne Hu. Structure Formation with Generalized Dark Matter. Astrophys. J., 506:485-494, 1998.

[24] D. J. Kaup. Klein-gordon geon. Phys. Rev., 172:1331, 1968.

[25] R. Ruffini and S. Bonazzola. System of self-gravitationg particles in general relativity and the concept of an equation of state. Phys. Rev., 187:1767-1783, 1969.

[26] P. Jetzer. Boson stars. Phys. Rep., 220:163-227, 1992. 
[27] F. E. Schunck and E. W. Mielke. General relativistic boson stars. Class. Quant. Grav., 20:R301-R356, 2003.

[28] Jae-weon Lee and In-gyu Koh. Galactic halos as boson stars. Phys. Rev., D53:2236-2239, 1996.

[29] T. D. Lee and Y. Pang. Stability of mini-boson stars. Nuclear Physics B, 315:477-516, March 1989.

[30] E. Seidel and W. Suen. Dynamical evolution of boson stars: Perturbing the ground state. Phys. Rev., D42:384, 1990.

[31] Tonatiuh Matos and L.Arturo Urena-Lopez. Flat rotation curves in scalar field galaxy halos. Gen.Rel.Grav., 39:1279-1286, 2007.

[32] Argelia Bernal, Juan Barranco, Daniela Alic, and Carlos Palenzuela. Multi-state Boson Stars. Phys.Rev., D81:044031, 2010.

[33] L.Arturo Urena-Lopez and Argelia Bernal. Bosonic gas as a Galactic Dark Matter Halo. Phys.Rev., D82:123535, 2010.

[34] I. Pena and D. Sudarsky. Do collapsed boson stars result in new types of black holes? Class. Quant. Grav., 14:3131-3134, 1997.

[35] J. D. Bekenstein. Novel 'no scalar hair' theorem for black holes. Phys. Rev., D51:6608-6611, 1995.

[36] Ulises Nucamendi and Marcelo Salgado. Scalar hairy black holes and solitons in asymptotically flat space-times. Phys.Rev., D68:044026, 2003.

[37] R. L. Marsa and M. W. Choptuik. Black hole scalar field interactions in spherical symmetry. Phys. Rev., D54:4929-4943, 1996.

[38] Ted Jacobson. Primordial black hole evolution in tensor scalar cosmology. Phys.Rev.Lett., 83:2699-2702, 1999.

[39] Rachel Bean and Joao Magueijo. Could supermassive black holes be quintessential primordial black holes? Phys.Rev., D66:063505, 2002.

[40] Andrei V. Frolov. Accretion of ghost condensate by black holes. Phys. Rev., D70:061501, 2004 .

[41] E. Babichev, V. Dokuchaev, and Yu. Eroshenko. Black hole mass decreasing due to phantom energy accretion. Phys.Rev.Lett., 93:021102, 2004. 
[42] E. Babichev, V. Dokuchaev, and Yury Eroshenko. The Accretion of dark energy onto a black hole. J.Exp.Theor.Phys., 100:528-538, 2005.

[43] L. Arturo Urena-Lopez and Lizbeth M. Fernandez. Black holes and the absorption rate of cosmological scalar fields. 2011.

[44] Luis Arturo Urena-Lopez and Andrew R. Liddle. Supermassive black holes in scalar field galaxy halos. Phys. Rev., D66:083005, 2002.

[45] A. Cruz-Osorio, F. S. Guzman, and F. D. Lora-Clavijo. Scalar Field Dark Matter: behavior around black holes. JCAP, 1106:029, 2011.

[46] Miguel Megevand, Ignacio Olabarrieta, and Luis Lehner. Scalar field confinement as a model for accreting systems. Class. Quant. Grav., 24:3235-3258, 2007.

[47] C. W. Misner, K. S. Thorne, and J. A. Wheeler. Gravitation. Academic Press, San Francisco, 1973.

[48] B.P. Jensen and P. Candelas. The Schwarzschild radial functions. Phys.Rev., D33:1590, 1986.

[49] A. Zecca. Properties of radial equation of scalar field in Schwarzschild space-time. Nuovo Cim., 124BN12:1251-1258, 2009.

[50] Claude Amsler et al. Review of particle physics. Phys. Lett., B667:1-1340, 2008.

[51] W. H. Press, B. P. Flannery, S. A. Teukolsky, and W. T. Vetterling. Numerical Recipes. Cambridge University Press, Cambridge, England, 1986.

[52] Juan Carlos Degollado, Dario Nunez, and Carlos Palenzuela. Signatures of the sources in the gravitational waves of a perturbed Schwarzschild black hole. Gen. Rel. Grav., 42:1287-1310, 2010. 